\title{
OPTIMAL ERROR ESTIMATES OF THE DIRECT DISCONTINUOUS GALERKIN METHOD FOR CONVECTION-DIFFUSION EQUATIONS
}

\author{
HAILIANG LIU
}

\begin{abstract}
In this paper, we present the optimal $L^{2}$-error estimate of $O\left(h^{k+1}\right)$ for polynomial elements of degree $k$ of the semidiscrete direct discontinuous Galerkin method for convection-diffusion equations. The main technical difficulty lies in the control of the inter-element jump terms which arise because of the convection and the discontinuous nature of numerical solutions. The main idea is to use some global projections satisfying interface conditions dictated by the choice of numerical fluxes so that trouble terms at the cell interfaces are eliminated or controlled. In multi-dimensional case, the orders of $k+1$ hinge on a superconvergence estimate when tensor product polynomials of degree $k$ are used on Cartesian grids. A collection of projection errors in both one- and multi-dimensional cases is established.
\end{abstract}

\section{INTRODUCTION}

In this article, we introduce an approach for proving optimal $L^{2}$-error estimates for the semidiscrete direct discontinuous Galerkin (DDG) method solving convection-diffusion problems. To demonstrate the main idea, we will focus on the one-dimensional model equations

$$
\partial_{t} U+\partial_{x} f(U)=\partial_{x}^{2} U
$$

with zero flux $f=0$, linear flux $f=\alpha U$ and nonlinear smooth flux $f(U)$, followed by multi-dimensional extensions. The DDG method was introduced in [27, refined with interface corrections in [28, and has since been extended to multi-dimensional settings as well as equations with nonlinear diffusion, for which extensive numerical tests have shown the optimal $(k+1)$ th order of accuracy for polynomial elements of degree $k$. However, the optimal $L^{2}$-error estimate has not been available. In this work we present a novel approach to obtain such an estimate. We will give the details of the proof for linear diffusion with different convections to illustrate the main ideas.

The discontinuous Galerkin (DG) method we discuss in this paper is a class of finite element methods, using a completely discontinuous piecewise polynomial space for the numerical solution and the test functions. One main advantage of the DG method was the flexibility afforded by local approximation spaces combined with the suitable design of numerical fluxes crossing cell interfaces. It was first designed and has been quite successful for solving first order PDEs such as hyperbolic

Received by the editor February 19, 2013 and, in revised form, August 13, 2013 and December $12,2013$.

2010 Mathematics Subject Classification. Primary 35K15, 65M15, 65M60, 76R50.

Key words and phrases. Convection-diffusion equations, discontinuous Galerkin, global projection, numerical flux. 
conservation laws [15, 17, 18, 21, 31]. However, the application of the DG method to diffusion problems has been a challenging task because of the subtle difficulty in defining appropriate numerical fluxes for the solution gradient. There have been several DG methods suggested in the literature to solve the problem, including the method originally proposed by Bassi and Rebay [5] for compressible Navier-Stokes equations, its generalization called the local discontinuous Galerkin (LDG) methods introduced in [19] by Cockburn and Shu and further studied in [8, 10, 14, 16], as well as the method introduced by Baumann-Oden 6, 29. See also the earlier works [1, 3, 35] using the so-called interior penalty (IP) method, and the unified analysis of DG methods in [2] for elliptic problems and background references for the IP methods.

The idea of DDG methods for higher order partial differential equations, such as the convection diffusion equation (11), is to directly force the weak solution formulation of the PDE into the DG function space for both the numerical solution and test functions. Unlike the traditional LDG method, the DDG method does not introduce any auxiliary variables or rewrite the original equation into a larger first order system. A key ingredient for the success of such methods is the correct interface corrections. These corrections must be selected to guarantee stability and solvability of the unknown to approximate the solution. The main novelty in the DDG schemes proposed in [27,28, lies in numerical flux choices for the solution gradient, which involves high order derivatives evaluated on cell interfaces, motivated by a trace formula for the solution derivatives of the heat equation [27]. With this choice, the obtained schemes are provably stable and optimally convergent. There are other recent works also featuring the direct DG discretization, such as those by van Leer and Nomura in [25, Gassner et al. in [22], and Cheng and Shu in [20]. Stability and convergence of the schemes are presented in [20] which takes advantage of some carefully designed numerical fluxes.

Obtaining a priori error estimates for various DG methods has been a main subject of research. For smooth solutions of scalar conservation laws, error estimates have been given in several earlier works [24, 26, 30, 32] and the more recent one [40] for the fully discrete Runge-Kutta DG methods, and [41] for symmetrizable systems. The first a priori error estimate of order $O\left(h^{k}\right)$ for the LDG method of linear convection-diffusion was obtained in [19. With a particular numerical flux, the optimal convergence rate of order $O\left(h^{k+1}\right)$ was obtained in [7, 10,11]. For the numerical method of Baumann and Oden [6] when applied to nonlinear convectiondiffusion equations, the optimal error estimate for at least quadratic polynomials was obtained by Riviere and Wheeler [33. The $L^{2}$ a priori error estimates for nonlinear PDEs with high order derivatives such as the KdV equations have been obtained [20, 36, 38, using certain special local projections. The optimal $L^{2}$-error estimate for the linearized $\mathrm{KdV}$ equation was obtained in [37, where authors take advantage of stability estimates for auxiliary variables. A conservative discontinuous Galerkin-method for the generalized $\mathrm{KdV}$ equation was recently proposed by Bona et al. 4, in which a global projection was used in obtaining error estimates in some cases.

For the DDG method, the first a priori error estimate of order $O\left(h^{k}\right)$ for linear diffusion was obtained in [28]. The accurate recovery algorithm of the normal derivatives presented in 23] provides a set of effective choices of parameters in the DDG numerical fluxes. An accuracy analysis using Fourier modes for some special 
solutions was presented in 39. The main objective of this article is to present an approach to obtain the optimal a priori error estimate of order $O\left(h^{k+1}\right)$ for polynomial elements of degree $k$.

In this paper, the main procedure to obtain the a priori error estimates is the following. First, we obtain the error equation for the DDG method. Second, we introduce a new global projection on $P U: \int_{I_{j}}(U-P U) v d x=0$ for any $v \in P^{k-2}$ subject to interface conditions dictated by the DDG numerical fluxes, and prove the existence of such a projection and obtain the projection error. Third, we split the error into two parts by using the global projections: $u-P U$ and $P U-U$, which enables us to control both cell integrals and the inter-element jump terms simultaneously. In the multi-dimensional case with nonlinear convection, we use tensor product polynomials of degree at most $k$ and show that the optimal error estimate of order $k+1$ for $k \geq \frac{d-1}{2}$; this restriction is unnecessary for the linear convection. In multi-dimensional case, a superconvergence result is established by taking advantage of the Cartesian structure of the grid and a similar argument to that in 9] for the LDG method to solve elliptic problems on Cartesian grids.

The paper is organized as follows. In $\S 2$ we illustrate a natural way to derive the global version of the DG formulation from the PDE weak solution formulation. We further discuss how a careful choice of numerical fluxes can be made to ensure some desired features including consistency, conservation, stability and accuracy. In $\S 3$, we quantify the admissible set of numerical fluxes for the solution gradient and the convection to ensure the $L^{2}$ stability. In $\S 4$ we estimate the $L^{2}$-error of the numerical solution from the original solution for the case of purely linear diffusion and linear convection-diffusion with two different global projections. In $\S 5$ we present the $L^{2}$-error estimate for linear diffusion with nonlinear convection. The extension to multi-dimensions is given is $\S 6$. A collection of projection errors in both one and multi-dimensional cases is presented in $\S 7$.

\section{Scheme FORMUlation}

We begin with the one-dimensional convection diffusion equation

$$
\partial_{t} U+\partial_{x} f(U)-\partial_{x}^{2} U=0
$$

subject to initial data

$$
U(x, 0)=U_{0}(x)
$$

posed on $\Omega:=[0,1]$ with periodic boundary conditions. The weak solution formulation for this problem is to find a function $U \in C\left(0, T ; H^{1}(\Omega)\right)$ such that for all $v \in H_{0}^{1}(\Omega)$,

$$
\begin{aligned}
& \left\langle\partial_{t} U, v\right\rangle-\left\langle f(U), \partial_{x} v\right\rangle+\left\langle\partial_{x} U, \partial_{x} v\right\rangle=0, \forall v \in H_{0}^{1}(\Omega), \\
& \langle U(x, 0), v\rangle=\left\langle U_{0}, v\right\rangle .
\end{aligned}
$$

Here $\langle\cdot, \cdot\rangle$ denotes the inner product of two functions over $\Omega$. To discretize this weak formulation, we set up a partition of the domain $\Omega=\bigcup_{j=1}^{N} I_{j}$, with mesh $I_{j}=\left[x_{j-1 / 2}, x_{j+1 / 2}\right]$ and mesh size $h:=\Delta x=x_{j+1 / 2}-x_{j-1 / 2}$, and define the finite element space

$$
\mathbb{V}_{h}^{k}:=\left\{v \in L^{2}(\Omega):\left.\quad v\right|_{I_{j}} \in P^{k}\left(I_{j}\right), \quad j=1, \ldots, N\right\},
$$


where $P^{k}\left(I_{j}\right)$ denotes the space of polynomials on $I_{j}$ with degree at most $k$. We will also adopt the following notation:

$$
u^{ \pm}=u(x \pm 0, t), \quad[u]=u^{+}-u^{-}, \quad\{u\}=\frac{u^{+}+u^{-}}{2} .
$$

The idea of the direct discontinuous Galerkin (DDG) method in [28] is to enforce the weak formulation (4), (5) in such a way that both $u$ and $v$ are approximated in $\mathbb{V}_{h}^{k}$. The discontinuous nature of numerical solutions and test functions crossing interfaces necessarily requires some interface corrections, leading to the following:

$$
\begin{gathered}
\sum_{j=1}^{N} \int_{I_{j}} \partial_{t} u v d x+\sum_{j=1}^{N}\left(-\int_{I_{j}} f(u) \partial_{x} v d x+\int_{I_{j}} \partial_{x} u \partial_{x} v d x\right) \\
+\sum_{j=1}^{N}\left(\left(-\hat{f}+\widehat{\partial_{x} u}\right)[v]+\widehat{\partial_{x} v}[u]\right)_{j+1 / 2}=0 \\
\sum_{j=1}^{N} \int_{I_{j}} u(x, 0) v d x=\sum_{j=1}^{N} \int_{I_{j}} U_{0} v d x
\end{gathered}
$$

Here, periodicity is realized by using same polynomials in $I_{1}$ as in $I_{N+1}$, and the "hat" terms are determined as numerical fluxes. Crucial for the scheme stability as well as for the accuracy of the DG method is the choice of numerical fluxes. The guiding principle is that numerical fluxes are chosen in such a way that they depend only on the left and right polynomials and that

(i) they are consistent with $-f(u)+\partial_{x} u$ when $u$ is smooth;

(ii) they are conservative in the sense that they are single valued on $x_{j+1 / 2}$,

(iii) they ensure stability, and

(iv) they enforce the high order accuracy of the method.

For convection we take the numerical flux

$$
\hat{f}=\hat{f}\left(u^{-}, u^{+}\right),
$$

which is Lipschitz continuous in its arguments, and consistent with $f$ in the sense that $\hat{f}(u, u)=f(u)$, and satisfies

$$
\int_{u^{-}}^{u^{+}}\left(\hat{f}\left(u^{+}, u^{-}\right)-f(u)\right) d u=\left(\hat{f}\left(u^{+}, u^{-}\right)-\frac{\int_{u^{-}}^{u^{+}} f(u) d u}{[u]}\right)[u] \leq 0 .
$$

This corresponds to the entropy flux with only quadratic entropy, and it may be called the quadratic entropy flux.

For diffusion, following [28], we take $\widehat{\partial_{x} v}=\left\{\partial_{x} v\right\}$ and

$$
\widehat{\partial_{x} u}=\frac{\beta_{0}}{h}[u]+\left\{\partial_{x} u\right\}+\beta_{1} h\left[\partial_{x}^{2} u\right]
$$

The algorithm is now well defined once the two parameters $\beta_{i}$ are chosen, and a particular $\hat{f}$ is selected. 
If we define two operators as

$$
\begin{aligned}
& A(u, v)=\sum_{j=1}^{N} \int_{I_{j}} \partial_{x} u \partial_{x} v d x+\left.\sum_{j=1}^{N}\left(\widehat{\partial_{x} u}[v]+[u]\left\{\partial_{x} v\right\}\right)\right|_{j+\frac{1}{2}}, \\
& F(u, v)=\sum_{j=1}^{N} \int_{I_{j}} f(u) \partial_{x} v d x+\left.\sum_{j=1}^{N} \hat{f}[v]\right|_{j+\frac{1}{2}},
\end{aligned}
$$

then the scheme may be compactly written as

$$
\left\langle\partial_{t} u, v\right\rangle+A(u, v)=F(u, v), \quad \forall v \in \mathbb{V}_{h}^{k} .
$$

This formulation is particularly convenient to analyze the scheme.

Restrict $v$ to only one cell $I_{j}$ and we may obtain the local version

$$
\int_{I_{j}} \partial_{t} u v d x=\int_{I_{j}}\left(f(u)-\partial_{x} u\right) \partial_{x} v d x+\left.\left[\left(-\hat{f}+\widehat{\partial_{x} u}\right) v+(u-\hat{u}) \partial_{x} v\right]\right|_{\partial I_{j}},
$$

with $\hat{u}=\{u\}, \widehat{\partial_{x} u}$ defined in (10) and $\hat{f}$ in (8).

We end this section by some further discussions on choices of the numerical flux $\hat{f}$. After Osher [42, a numerical flux may be called the entropy flux if it satisfies

$$
\int_{u^{-}}^{u^{+}} \eta_{u u}\left(\hat{f}\left(u^{+}, u^{-}\right)-f(u)\right) d u \leq 0
$$

for any convex entropy function $\eta$, for it is equivalent to the E-flux as defined by

$$
\operatorname{sign}\left(u^{+}-u^{-}\right)\left(\hat{f}\left(u^{+}, u^{-}\right)-f(u)\right) \leq 0
$$

for all $u$ between $u^{-}$and $u^{+}$. A popular subclass is the so-called monotone fluxes for which $\hat{f}$ is nondecreasing in $u^{-}$and nonincreasing in $u^{+}$. A well-known monotone flux is the Lax-Friedrich flux of the form

$$
\hat{f}=\{f(u)\}-\frac{\sigma}{2}[u], \quad \sigma=\max \left|f^{\prime}\right| .
$$

Such a choice will dissipate the entropy, and be particularly suitable for discontinuous solutions or solutions with sharp fronts when convection dominates. To summarize the relation between the different types of fluxes considered above, we have

monotone flux (16) $\Rightarrow$ entropy flux (15) $\Rightarrow$ quadratic entropy flux (9) .

In other words, examples of monotone and entropy fluxes are actually also the quadratic entropy flux (91). In the presence of diffusion, one may take

$$
\hat{f}=\frac{\int_{u^{-}}^{u^{+}} f(u) d u}{[u]}=: \underset{u^{-}}{u^{+}} f(u) d u
$$

which is a quadratic entropy flux.

For any piecewise smooth function $u \in L^{2}$, on any cell interface we define

$$
\alpha(\hat{f} ; \xi):= \begin{cases}{[u]^{-1}(f(\xi)-\hat{f}),} & \forall \xi \in\left(\min \left(u^{-}, u^{+}\right), \max \left(u^{-}, u^{+}\right)\right), \\ \frac{1}{2} \max \left|f^{\prime}\right|, & \text { if }[u]=0,\end{cases}
$$


where $\hat{f} \equiv \hat{f}\left(u^{-}, u^{+}\right)$is an entropy satisfying flux consistent with the given flux $f(u)$. We then find that for the quadratic entropy flux (9),

$$
\alpha\left(\hat{f}, u^{*}\right) \geq 0, \quad f\left(u^{*}\right)=\underset{u^{-}}{u^{+}} f(u) d u,
$$

and for monotone fluxes we have

$$
\alpha(\hat{f}, \xi) \geq 0, \quad \forall \xi \in\left(\min \left(u^{-}, u^{+}\right), \max \left(u^{-}, u^{+}\right)\right) .
$$

Notations. Throughout the paper we use unmarked norm $\|\cdot\|_{B}$ or $\|\cdot\|_{0, B}$ as the $L^{2}$-norm on domain $B,\|\cdot\|_{m, B}$ as the $H^{m}$-norm and $|\cdot|_{m, B}$ as the seminorm of $H^{m}$ defined by $|u|_{m, B}=\left(\sum_{|\alpha|=m} \int_{B}\left|\partial_{x}^{\alpha} u\right|^{2} d x\right)^{1 / 2}$. We use $\|\cdot\|_{\infty, B}$ to denote the $L^{\infty}$-norm. $B$ may not be included in the norm expression if it is the whole domain or the domain is clear in the context.

\section{NUMERICAL FLUX AND $L^{2}$ STABILITY}

We define the discrete energy norm by

$$
\|v\|_{E}^{2}=\sum_{j=1}^{N} \int_{I_{j}}\left|\partial_{x} v\right|^{2} d x+\left.\sum_{j=1}^{N} \frac{\beta_{0}}{h}[v]^{2}\right|_{j+\frac{1}{2}}, \quad v \in \mathbb{V}_{h}^{k},
$$

and introduce a quantity

$$
\Gamma\left(\beta_{1}\right)=\sup _{v \in P^{k-1}[-1,1]} \frac{\left(v(1)-2 \beta_{1} \partial_{\xi} v(1)\right)^{2}}{f_{-1}^{1} v^{2}(\xi) d \xi},
$$

where $\xi$ may be interpreted as $2\left(x-x_{j}\right) / \Delta x$ for $x \in I_{j}$. It is clear that $\Gamma$ depends on both $\beta_{1}$ and the polynomial degree $k$. Note that, for any $v_{1}, v_{2} \in P^{k-1}([-1,1])$ and $v=v_{1}+v_{2}$, we have

$$
\left(v(1)-2 \beta_{1} \partial_{\xi} v(1)\right)^{2} \leq \frac{\Gamma}{2} \int_{-1}^{1} v^{2}(\xi) d \xi \leq \Gamma\left(\int_{-1}^{1}\left|v_{1}(\xi)\right|^{2} d \xi+\int_{-1}^{1}\left|v_{2}(\xi)\right|^{2} d \xi\right) .
$$

This, when applied to the numerical solution $u$ with

$$
v_{1}(\xi)=v_{1}\left(\frac{x-x_{j}}{h / 2}\right)=\left.\partial_{x} u\right|_{I_{j}}, \quad v_{2}(\xi)=v_{2}\left(\frac{x_{j+1}-x}{h / 2}\right)=\left.\partial_{x} u\right|_{I_{j+1}},
$$

gives

$$
\left(2\left\{\partial_{x} u\right\}+\beta_{1} h\left[\partial_{x}^{2} u\right]\right)^{2} \leq \frac{2 \Gamma}{h}\left(\int_{I_{j}}\left|\partial_{x} u\right|^{2} d x+\int_{I_{j+1}}\left|\partial_{x} u\right|^{2} d x\right), \quad u \in \mathbb{V}_{h}^{k} .
$$

Summing over $j=1, \ldots, N$ we obtain

$$
h \sum_{j=1}^{N}\left(\left\{\partial_{x} u\right\}+\frac{\beta_{1}}{2} h\left[\partial_{x}^{2} u\right]\right)^{2} \leq \Gamma \sum_{j=1}^{N} \int_{I_{j}}\left|\partial_{x} u\right|^{2} d x .
$$

Here we have used $\left.u\right|_{I_{1}}=\left.u\right|_{I_{N+1}}$ to incorporate the periodic boundary condition. This implies that

$$
\Gamma \geq \sup _{u \in P^{k}\left(I_{j}\right)} \frac{h \sum_{j=1}^{N}\left(\left\{\partial_{x} u\right\}+\frac{\beta_{1}}{2} h\left[\partial_{x}^{2} u\right]\right)^{2}}{\sum_{j=1}^{N} \int_{I_{j}}\left|\partial_{x} u\right|^{2} d x} .
$$


The right hand side is the lower bound for $\beta_{0}$ obtained in [28, using the admissibility criteria. We thus conclude that there exists $\gamma \in(0,1)$ such that

$$
A(v, v) \geq \gamma\|v\|_{E}^{2}, \quad \forall v \in \mathbb{V}_{h}^{k}
$$

provided

$$
\beta_{0}>\Gamma\left(\beta_{1}\right) .
$$

We next present a simple evaluation of $\Gamma$.

Lemma 3.1. For any $k \geq 1$, it holds that

$$
\Gamma\left(\beta_{1}\right)=k^{2}\left(1-\beta_{1}\left(k^{2}-1\right)+\frac{\beta_{1}^{2}}{3}\left(k^{2}-1\right)^{2}\right) .
$$

Moreover, $\Gamma\left(\beta_{1}\right)$ achieves its minimum $\frac{k^{2}}{4}$ at $\beta_{1}^{*}=\frac{3}{2\left(k^{2}-1\right)}$.

Proof. Let $\psi(\xi)$ be a vector basis function, satisfying $\int_{-1}^{1} \psi_{i}(\xi) \psi_{l}(\xi) d \xi=\delta_{i l}$ for $1 \leq i, l \leq k$, and let $v(\xi)=\sum_{i=1}^{k} a_{i} \psi_{i}(\xi)$, then

$$
\int_{-1}^{1}|v|^{2} d \xi=a^{T}\left(\int_{-1}^{1} \psi \psi^{T} d \xi\right) a=\sum_{j=1}^{k}\left|a_{j}\right|^{2}=|a|^{2} .
$$

On the other hand, for any number $\gamma$,

$\left(v(1)+\gamma \partial_{\xi} v(1)\right)^{2}=a^{T}\left(\psi(1)+\gamma \partial_{\xi} \psi(1)\right)\left(\psi(1)+\gamma \partial_{\xi} \psi(1)\right)^{T} a \leq\left|\psi(1)+\gamma \partial_{\xi} \psi(1)\right|^{2}|a|^{2}$.

Hence for $\gamma=-2 \beta_{1}$ as in (22) we may take

$$
\Gamma\left(\beta_{1}\right)=2\left|\psi(1)+\gamma \partial_{\xi} \psi(1)\right|^{2} .
$$

To make this bound more precise, we choose the normalized Legendre polynomial vector basis $\left\{\psi_{i}\right\}_{i=1}^{k}$. Using

$$
\psi_{i}(1)=\sqrt{\frac{2 i-1}{2}}, \quad \partial_{\xi} \psi_{i}(1)=\sqrt{\frac{2 i-1}{2}} \frac{i(i-1)}{2}, \quad i=1, \ldots, k,
$$

we obtain for any $\gamma \in \mathbb{R}$,

$$
\begin{aligned}
2\left|\psi(1)+\gamma \partial_{\xi} \psi(1)\right|^{2} & =2 \sum_{i=1}^{k} \frac{2 i-1}{2}\left|1+\frac{\gamma}{2} i(i-1)\right|^{2} \\
& =\sum_{i=1}^{k}(2 i-1)+\gamma \sum_{i=1}^{k-1} i(i+1)(2 i+1)+\frac{\gamma^{2}}{4} \sum_{i=1}^{k-1} i^{2}(i+1)^{2}(2 i+1) \\
& =k^{2}\left(1+\frac{\gamma}{2}\left(k^{2}-1\right)+\frac{\gamma^{2}}{12}\left(k^{2}-1\right)^{2}\right)
\end{aligned}
$$

The desired result follows by taking $\gamma=-2 \beta_{1}$. Particularly, it achieves the minimum $\frac{k^{2}}{4}$ at $\gamma^{*}=-\frac{3}{k^{2}-1}$.

Lemma 3.2. For the quadratic entropy flux $\hat{f}$ defined in (9), it holds that

$$
F(u, u) \leq 0 .
$$


Proof. Using the periodic boundary condition, we calculate

$$
\begin{aligned}
& \sum_{j=1}^{N} \int_{I_{j}} f(u) \partial_{x} u d x \\
&=-\sum_{j=1}^{N}\left(f\left(u^{*}\right)[u]\right)_{j+1 / 2} \quad \text { with } f\left(u^{*}\right)={\underset{u}{u^{-}}}_{u^{+}}^{f} f(u) d u .
\end{aligned}
$$

Hence for quadratic entropy flux (9),

$$
\begin{aligned}
F(u, u) & =\sum_{j=1}^{N}\left(\hat{f}\left(u^{-}, u^{+}\right)[u]\right)_{j+1 / 2}-\sum_{j=1}^{N}\left(f\left(u^{*}\right)[u]\right)_{j+1 / 2} \\
& =-\sum_{j=1}^{N}\left(\alpha\left(\hat{f}, u^{*}\right)[u]^{2}\right)_{j+1 / 2} \leq 0 .
\end{aligned}
$$

This lemma and (24) ensures that the DDG scheme (13) has the following provable properties.

Theorem 3.3. Consider the semidiscrete $D G$ (13) with $\left(\beta_{0}, \beta_{1}\right)$ satisfying (25) and the quadratic entropy flux (9) for convection, then it satisfies the following properties:

(1) Conservation of mass: $\sum_{j=1}^{N} \int_{I_{j}} u(t, x) d x=\int_{\Omega} U_{0}(x) d x, \quad \forall t>0$.

(2) The energy $\|u\|^{2}:=\sum_{j=1}^{N} \int_{I_{j}} u^{2} d x$ is nonincreasing in time. More precisely, there exists $\gamma \in(0,1)$ such that

$$
\frac{d}{d t}\|u\|^{2} \leq-2 \gamma\|u\|_{E}^{2} \leq 0 .
$$

(3) The scheme is $L^{2}$ stable in the sense that

$$
\|u\|^{2} \leq \int_{\Omega} U_{0}^{2} d x, \quad \forall t>0 .
$$

Proof. (1) Taking $v=1$ in (13) we have $\frac{d}{d t} \sum_{j=1}^{N} \int_{I_{j}} u d x=0$, which when combined with (7) with $v=1$ leads to the mass conservation.

(2) Take $v=u$ in (13) and we obtain

$$
\frac{1}{2} \frac{d}{d t}\|u\|^{2}+A(u, u)=F(u, u) .
$$

For the quadratic entropy flux, we have

$$
F(u, u) \leq 0 .
$$

This together with the lower bound of $A$ ensures the energy dissipation property (28).

(3) From (2) it follows that

$$
\|u\|^{2} \leq\|u(\cdot, 0)\|^{2}=\sum_{j=1}^{N} \int_{I_{j}} u^{2}(x, 0) d x \leq \sum_{j=1}^{N} \int_{I_{j}} U_{0}^{2} d x
$$

by using (7). 


\section{ERror estimates}

The idea for obtaining the optimal error estimate is to introduce some special global projection. We illustrate this idea for diffusion first, and then discuss its extension to convection-diffusion equations.

4.1. A global projection and projection error. We define a special projection $P$ as follows. For a given smooth function $w$, the projection $P w$ is the unique function in $\mathbb{V}_{h}^{k}$ which satisfies

$$
\begin{aligned}
& \int_{I_{j}}(P w(x)-w(x)) v(x) d x=0, \quad \forall v \in P^{k-2}\left(I_{j}\right), j=1, \ldots, N \\
& \widehat{\partial_{x}(P w)}:=\beta_{0} h^{-1}[P w]+\left\{\partial_{x}(P w)\right\}+\left.\beta_{1} h\left[\partial_{x}^{2}(P w)\right]\right|_{j+1 / 2}=\partial_{x} w\left(x_{j+1 / 2}\right) \\
& \left.\{P w\}\right|_{j+1 / 2}=w\left(x_{j+1 / 2}\right)
\end{aligned}
$$

At $x_{N+1 / 2}$ we use a periodic extension to be consistent with the periodic boundary condition for problems (2) and (3). For a piecewise smooth function $w$ with $\left.w\right|_{I_{j}} \in$ $H^{k+1}\left(I_{j}\right)$, the above definition needs to be modified so that the right of (29) $\left.\mathrm{b}\right)$ and (29k) is replaced by $\widehat{w_{x}}$ and $\{w\}$, respectively.

Lemma 4.1. For $\left(\beta_{0}, \beta_{1}\right)$ such that $\beta_{0}>\Gamma\left(\beta_{1}\right)$, the projection $P$ defined in (29) exists, and

$$
\left(\int_{0}^{1}(P w(x)-w(x))^{2} d x\right)^{1 / 2} \leq C h^{k+1}
$$

for a smooth function $w$, where $C$ is a constant depending on $w$ and its derivatives but independent of $h$.

Proof. We first show the existence of such a projection. Define the affine map

$$
x=x(\xi)=x_{j}+\frac{h}{2} \xi,-1 \leq \xi \leq 1,
$$

which maps $[-1,1]$ onto $I_{j}$. The Legendre polynomials $\phi_{k}\left(2\left(x-x_{j}\right) / h\right)=L_{k-1}(\xi)$ are the Legendre polynomials on $[-1,1]$, then the projection can be expressed in terms of the Legendre polynomials

$$
\left.P w(x)\right|_{I_{j}}=\sum_{i=1}^{k+1} a_{i}^{j} \phi_{i}(\xi), \quad 1 \leq j \leq N .
$$

From (29) with $v=\left\{\phi_{i}\right\}_{i=1}^{k-1}$ it follows that for $1 \leq j \leq N$,

$$
a_{i}^{j}=\frac{2 i-1}{2} \int_{-1}^{1} w\left(x_{j}+\frac{h}{2} \xi\right) \phi_{i}(\xi) d \xi, \quad i=1, \ldots, k-1,
$$

where we have used $\left\|\phi_{i}\right\|^{2}=\frac{2}{2 i-1}$. It remains to determine $a_{k}^{j}$ and $a_{k+1}^{j}$ by using the interface conditions; that is, $\left.\{P w\}\right|_{j+1 / 2}=w\left(x_{j+1 / 2}\right)$ and

$$
\beta^{0}[P w]+2\left\{\partial_{\xi}(P w)\right\}+4 \beta_{1}\left[\partial_{\xi}^{2}(P w)\right]=h \partial_{x} w\left(x_{j+1 / 2}\right),
$$


which lead to

$$
\begin{gathered}
\sum_{i=k}^{k+1}\left[\phi_{i}(1) a_{i}^{j}+\phi_{i}(-1) a_{i}^{j+1}\right]=b_{1}^{j}:=2 w\left(x_{j+1 / 2}\right)-\sum_{i=1}^{k-1}\left[\phi_{i}(1) a_{i}^{j}+a_{i}^{j+1} \phi_{i}(-1)\right], \\
\sum_{i=k}^{k+1}\left[g_{0}(i) a_{i}^{j}+g_{1}(i) a_{i}^{j+1}\right]=b_{2}^{j}:=h w_{x}\left(x_{j+1 / 2}\right)-\sum_{i=1}^{k-1}\left[g_{0}(i) a_{i}^{j}+g_{1}(i) a_{i}^{j+1}\right],
\end{gathered}
$$

where

$$
g_{0}(i)=-\beta_{0} \phi_{i}(1)+\phi_{i}^{\prime}(1)-4 \beta_{1} \phi_{i}^{\prime \prime}(1), \quad g_{1}(i)=\beta_{0} \phi_{i}(-1)+\phi_{i}^{\prime}(-1)+4 \beta_{1} \phi_{i}^{\prime \prime}(-1) .
$$

Set two matrices

$$
A=\left(\begin{array}{cc}
\phi_{k}(1) & \phi_{k+1}(1) \\
g_{0}(k) & g_{0}(k+1)
\end{array}\right), \quad B=\left(\begin{array}{cc}
\phi_{k}(-1) & \phi_{k+1}(-1) \\
g_{1}(k) & g_{1}(k+1)
\end{array}\right)
$$

then the coefficient matrix of $\left\{a^{j}\right\}_{j=1}^{N}$ with $a^{j}=\left(a_{k}^{j}, a_{k+1}^{j}\right)^{\top}$ is an $N \times N$ block circulant matrix with the first row $\left[\begin{array}{lllll}A & B & 0 & \cdots & 0\end{array}\right]$ and the last row $\left[\begin{array}{llllll}B & 0 & \cdots & 0 & A\end{array}\right]$, denoted by $D$. The determinant of this matrix is

$$
\operatorname{det} D=|A|^{N}\left|I+(-1)^{N-1}\left(A^{-1} B\right)^{N}\right|,
$$

which is nonzero as long as $|A \pm B| \neq 0$. Recall that, for $i \geq 1$, we have

$$
\begin{aligned}
\phi_{i}( \pm 1) & =( \pm 1)^{i-1}, i=1,2, \ldots, \\
\phi_{i}^{\prime}( \pm 1) & =\frac{1}{2}( \pm 1)^{i} i(i-1), i=2,3, \ldots, \\
\phi_{i}^{\prime \prime}( \pm 1) & =\frac{1}{8}( \pm 1)^{i-1}(i-2)(i-1) i(i+1), \quad i=3,4, \ldots
\end{aligned}
$$

hence,

$$
g_{0}(i)=-\beta_{0}+\frac{1}{2} i(i-1)-\frac{\beta_{1}}{2}(i-2)(i-1) i(i+1), \quad g_{1}(i)=(-1)^{i} g_{0}(i) .
$$

By a direct calculation we see that $|A \pm B| \neq 0$ is equivalent to the requirement that

$$
g_{0}(i)+(-1)^{i} g_{1}(i) \neq 0, \quad i=k, k+1
$$

that is, $g_{0}(i) \neq 0$ for $i=k, k+1$. Under this condition the determinant of the coefficient matrix will not vanish, thus is nonsingular. The existence of global projection (29) follows. In fact, for $\beta_{0}>\Gamma\left(\beta_{1}\right)$ we do have $g_{0}(i) \neq 0$ for $i=k, k+1$. This can be verified by a straightforward calculation, leading to

$$
g_{0}(k)<-\frac{1}{2} k(k+1), \quad g_{0}(k+1)<-\frac{1}{2} k(k-1)
$$

for $\beta_{0}>\Gamma\left(\beta_{1}\right)$.

The desired $L^{2}$-error estimate follows from the refined estimates stated in Lemma 4.2 below. 
Lemma 4.2 (The projection estimates). If $w$ is a smooth function satisfying $w \in$ $H^{k+1}(\Omega)$, then we have the following estimates:

$$
\begin{aligned}
& \sum_{j=1}^{N}\|P w-w\|_{0, I_{j}}^{2} \leq C h^{2 k+2}|w|_{k+1, \Omega}^{2}, \\
& \sum_{j=1}^{N}|P w-w|_{j+1 / 2}^{2} \leq C h^{2 k+1}|w|_{k+1, \Omega}^{2}, \\
& \sum_{j=1}^{N}|P w-w|_{\infty, I_{j}}^{2} \leq C h^{2 k}|w|_{k+1, \Omega}^{2},
\end{aligned}
$$

where $C$ depends on $k, \beta_{0}, \beta_{1}$ but is independent of $I_{j}$ and $w$.

These projection error estimates follow from those stated in Theorem 7.1, which is proved in $\S 7$.

4.2. Projection and inverse properties. Let $\epsilon=P U-U$ denote the projection error, then Lemma 4.2 implies the following inequality:

$$
\|\epsilon\|+h\|\epsilon\|_{\infty}+h^{1 / 2}\|\epsilon\|_{\Gamma_{h}} \leq C h^{k+1},
$$

where $\Gamma_{h}$ denotes the set of interface points of all cells $I_{j}$, and the constant $C$ depends on $k$ and $|U|_{k+1}$.

For any function $e \in \mathbb{V}_{h}^{k}$, the following inverse inequalities hold [13]:

$$
\begin{aligned}
& \left\|\partial_{x}^{l} e\right\| \leq C h^{-l}\|e\|, \\
& \|e\|_{\Gamma_{h}} \leq C h^{-1 / 2}\|e\|, \\
& \|e\|_{\infty} \leq C h^{-1 / 2}\|e\| .
\end{aligned}
$$

4.3. Error analysis. If we assume the exact solution of (2) is smooth, we can obtain optimal $L^{2}$-error estimates.

Theorem 4.3 (Diffusion without convection $f=0$ ). The solution $u$ to the semidiscrete DDG scheme (13) for problem (2)-(3) with $f=\alpha u$ which admits a smooth solution $U$ satisfies the following error estimate:

$$
\int_{0}^{1}(u(x, t)-U(x, t))^{2} d x \leq C h^{2(k+1)},
$$

where $C$ depends on $U$ and its derivatives but is independent of $h$.

Proof. The DG scheme can be written as

$$
\left\langle\partial_{t} u, v\right\rangle+A(u, v)=0
$$

for all $v \in \mathbb{V}_{h}^{k}$. The scheme consistency ensures that the exact solution $U$ of (2) also satisfies

$$
\left\langle\partial_{t} U, v\right\rangle+A(U, v)=0
$$

for all $v \in \mathbb{V}_{h}^{k}$. Subtracting (38) from (39) and using the linearity of $A$ with respect to its first arguments, we obtain the error equation

$$
\left\langle\partial_{t}(U-u), v\right\rangle+A(U-u, v)=0
$$

for all $v \in \mathbb{V}_{h}^{k}$. 
We now take $v=P U-u$ in the error equation (40), and denote

$$
e=P U-u, \quad \epsilon=P U-U,
$$

to obtain

$$
\left\langle\partial_{t} e, e\right\rangle+A(e, e)=\left\langle\partial_{t} \epsilon, e\right\rangle+A(\epsilon, e) .
$$

For the left hand side of (42), we use the $L^{2}$ stability estimate in Theorem 3.3 to obtain

$$
\left\langle\partial_{t} e, e\right\rangle+A(e, e) \geq \frac{1}{2} \frac{d}{d t} \int_{0}^{1} e^{2} d x+\gamma\|e\|_{E}^{2} .
$$

As to the right hand side of (42), we first write out all the terms in $A(\epsilon, e)$ :

$$
\begin{aligned}
A(\epsilon, e) & =\sum_{j=1}^{N} \int_{I_{j}} \partial_{x} \epsilon \partial_{x} e d x+\sum_{j=1}^{N-1}\left(\widehat{\partial_{x}} \epsilon[e]+\left\{\partial_{x} e\right\}[\epsilon]\right)_{j+1 / 2} \\
& =-\sum_{j=1}^{N} \int_{I_{j}} \partial_{x}^{2} e \epsilon d x+\sum_{j=1}^{N-1}\left(-\left[\partial_{x} e \epsilon\right]+\widehat{\partial_{x}} \epsilon[e]+\left\{\partial_{x} e\right\}[\epsilon]\right)_{j+1 / 2} \\
& =-\sum_{j=1}^{N} \int_{I_{j}} \partial_{x}^{2} e \epsilon d x+\sum_{j=1}^{N-1}\left(\widehat{\partial_{x}} \epsilon[e]-\{\epsilon\}\left[\partial_{x} e\right]\right)_{j+1 / 2} .
\end{aligned}
$$

Noticing the properties of the projection $P$, we have

$$
\int_{I_{j}} \partial_{x}^{2} e \epsilon d x=0
$$

because $\partial_{x}^{2} e$ are polynomials of degree at most $k-2$, and at $x_{j+1 / 2}$,

$$
\widehat{\partial_{x}} \epsilon=\widehat{\partial_{x}(P U)}-\partial_{x} U=0, \quad\{\epsilon\}=\{P U\}-U=0 .
$$

Therefore $A(\epsilon, e)=0$ and the right hand side of (42) becomes

$$
\left\langle\partial_{t} \epsilon, e\right\rangle \leq \frac{1}{2} \int_{0}^{1}\left(\partial_{t} \epsilon\right)^{2} d x+\frac{1}{2} \int_{0}^{1} e^{2} d x .
$$

Plugging (43) and (44) into the equality (42) and using the approximation result in Lemma 4.1, we obtain

$$
\frac{d}{d t} \int_{0}^{1} e^{2} d x+2 \gamma\|e\|_{E}^{2} \leq \int_{0}^{1} e^{2} d x+C h^{2 k+2} .
$$

The Gronwall inequality, the fact of the initial error (initial data is obtained by a standard $L^{2}$ projection),

$$
\left\|U_{0}-u(\cdot, 0)\right\| \leq C h^{k+1},
$$

and the approximation result in Lemma4.1 finally give us the desired error estimate. 
4.4. Linear convection. The same optimal error estimate can be obtained for the convection-diffusion equation with linear convection $f(u)=\alpha u$. We modify the projection as follows. For a given smooth function $w$, the projection $Q w$ is the unique function in $\mathbb{V}_{h}^{k}$ which satisfies

$$
\begin{aligned}
& \int_{I_{j}}(Q w(x)-w(x)) v(x) d x=0, \quad \forall v \in P^{k-2}\left(I_{j}\right), j=1, \ldots, N, \\
& \widehat{\partial_{x}(Q w)}-\hat{f}(w)=\partial_{x} w\left(x_{j+1 / 2}\right)-\alpha w\left(x_{j+1 / 2}\right), \\
& \{Q w\}=w\left(x_{j+1 / 2}\right),
\end{aligned}
$$

where at $x_{N+1 / 2}$ periodic extension of $Q w$ is applied. For piecewise smooth function $w$, a similar modification to that for (29) suffices.

Lemma 4.4. For $\left(\beta_{0}, \beta_{1}\right)$ such that $\beta_{0}>\Gamma\left(\beta_{1}\right)$ and any real number $\alpha$, the projection $Q$ defined in (45) exists, and

$$
\left(\int_{0}^{1}(Q w(x)-w(x))^{2} d x\right)^{1 / 2} \leq C h^{k+1}
$$

for a smooth function $w$, where $C$ is a constant depending on $w$ and its derivatives but independent of $h$.

Proof. The proof is entirely similar to that in Lemma 4.1, except for a modification of $g_{0}$ and $g_{1}$,

$$
\widetilde{g}_{0}(i)=g_{0}(i)-\frac{\alpha}{2} h, \quad \widetilde{g}_{1}(i)=(-1)^{i}\left(g_{0}(i)+\frac{\alpha}{2} h\right) .
$$

From (31) in the proof of Lemma 4.1 it follows that the projection $Q$ exists, provided

$$
\widetilde{g}_{0}(i)+(-1)^{i} \widetilde{g}_{1}(i) \neq 0, \quad i=k, k+1 .
$$

In fact, the above quantity gives

$$
\widetilde{g}_{0}(i)+(-1)^{i} \widetilde{g}_{1}(i)=2 g_{0}(i), \quad i=k, k+1 .
$$

Hence the result in Lemma 4.1 remains valid for the projection $Q$.

Theorem 4.5 (Diffusion with linear convection $f=\alpha u$ ). The solution $u$ to the semidiscrete DDG scheme (13) for problem (2)-(3) with $f=\alpha u$ which admits a smooth solution $U$ satisfies the following error estimate:

$$
\int_{0}^{1}(u(x, t)-U(x, t))^{2} d x \leq C h^{2(k+1)},
$$

where $C$ depends on $U$ and its derivatives but is independent of $h$.

Proof. Following the proof of Theorem 4.3, we set

$$
e=Q U-u, \quad \epsilon=Q U-U,
$$

to obtain

$$
\left(\partial_{t} e, e\right)+A(e, e)-F(e, e)=\left(\partial_{t} \epsilon, e\right)+A(\epsilon, e)-F(\epsilon, e) .
$$

For the left hand side of (49), we use the $L^{2}$ stability estimate and $F(e, e) \leq 0$ to obtain

$$
\left(\partial_{t} e, e\right)+A(e, e)-F(e, e) \geq \frac{1}{2} \frac{d}{d t} \int_{0}^{1} e^{2} d x+\gamma\|e\|_{E}^{2} .
$$


As to the right hand side of (49), we first write out all the terms in $A(\epsilon, e)-F(\epsilon, e)$

$$
\begin{aligned}
A(\epsilon, e)-F(\epsilon, e)= & -\sum_{j=1}^{N}\left(\int_{I_{j}} e_{x x} \epsilon+f(\epsilon) e_{x}\right) d x \\
& +\sum_{j=1}^{N-1}\left(\left(\hat{\epsilon}_{x}-\widehat{f(\epsilon)}\right)[e]-\{\epsilon\}\left[e_{x}\right]\right)_{j+1 / 2} \\
= & -\sum_{j=1}^{N} \int_{I_{j}} f(\epsilon) e_{x} d x
\end{aligned}
$$

by the properties of the projection $Q$. Therefore, the right hand side of (49) becomes

$$
\left(\partial_{t} \epsilon, e\right)+A(\epsilon, e)-F(\epsilon, e) \leq \frac{1}{2} \int_{0}^{1} \epsilon_{t}^{2} d x+\int_{0}^{1} e^{2} d x+\frac{\alpha^{2}}{2 \gamma} \int_{0}^{1} \epsilon^{2} d x+\frac{\gamma}{2}\|e\|_{E}^{2} .
$$

Plugging (50) and (51) into the equality (49) and using the approximation results in Lemma 4.4, we obtain

$$
\frac{d}{d t} \int_{0}^{1} e^{2} d x+\gamma\|e\|_{E}^{2} \leq \int_{0}^{1} e^{2} d x+C h^{2 k+2} .
$$

Again by the Gronwall inequality, the initial error and the approximation result in Lemma 4.4, we obtain the desired error estimate.

Remark 4.1. For nonlinear convection, we can still obtain the optimal error estimates, yet the above proof relying on a modified projection is no longer applicable. In the rest of this paper we present an approach to overcome the difficulty caused by the nonlinear convection, and the argument applies to linear convection as well.

\section{ERROR ESTIMATES WITH NONLINEAR CONVECTION}

Theorem 5.1. The solution $u$ to the semidiscrete DDG scheme (13) (using a monotone flux for $\hat{f}$ ) for problem (2), (3) with a smooth solution $U$ satisfies the following error estimate:

$$
\int_{0}^{1}(u(x, t)-U(x, t))^{2} d x \leq C h^{2(k+1)},
$$

where $C$ depends on $U$ and its derivatives but is independent of $h$.

Proof. Let $P U$ be defined in (29). Following the proof of Theorem 4.3, we set

$$
e=P U-u, \quad \epsilon=P U-U,
$$

to obtain

$$
\left\langle\partial_{t} e, e\right\rangle+A(e, e)=\left\langle\partial_{t} \epsilon, e\right\rangle+A(\epsilon, e)+H, \quad H:=F(U, e)-F(u, e) .
$$

Using the coercivity $A(e, e) \geq \gamma\|e\|_{E}^{2}$, and $A(\epsilon, e)=0$ due to the special projection, we have

$$
\frac{1}{2} \frac{d}{d t}\|e\|^{2}+\gamma\|e\|_{E}^{2} \leq \frac{1}{2} \int_{0}^{1} \epsilon_{t}^{2} d x+\frac{1}{2} \int_{0}^{1} e^{2} d x+H
$$


To estimate $H$ from above, we take the average of $u^{ \pm}$as a reference value $\{u\}$, hence

$$
\begin{aligned}
H= & \sum_{j=1}^{N} \int_{I_{j}}(f(U)-f(u)) e_{x} d x+\sum_{j=1}^{N}((f(U)-f(\{u\}))[e])_{j+\frac{1}{2}} \\
& +\sum_{j=1}^{N}((f(\{u\})-\hat{f}(u))[e])_{j+\frac{1}{2}},
\end{aligned}
$$

where we have taken the periodic boundary condition into account.

First we establish the estimate of the last term in (56) as

$$
H_{2} \leq \frac{\gamma}{3} \sum_{j=1}^{N} \beta_{0} \frac{[e]^{2}}{h}+C h^{2 k+2}
$$

In fact, using notation (18) and the fact that the exact solution is continuous with $[U]=0$ so that $[u]=[\epsilon]-[e]$, we obtain

$$
H_{2}=\sum_{j=1}^{N}(\alpha(\hat{f} ;\{u\})[u][e])_{j+\frac{1}{2}}=-\sum_{j=1}^{N}\left(\alpha(\hat{f} ;\{u\})[e]^{2}\right)_{j+\frac{1}{2}}+\sum_{j=1}^{N}(\alpha(\hat{f} ;\{u\})[\epsilon][e])_{j+\frac{1}{2}} .
$$

For the monotone flux, the first term is nonpositive; see (20). Using the Young inequality, we bound the last term further by

$$
\frac{\gamma}{3} \sum_{j=1}^{N} \beta_{0} \frac{[e]^{2}}{h}+\frac{3 h}{4 \beta_{0} \gamma} \sum_{j=1}^{N}\left(\alpha^{2}[\epsilon]^{2}\right)_{j+\frac{1}{2}} .
$$

The claimed bound for $\mathrm{H}_{2}$ follows from these estimates with the projection error inequality (30),

$$
\sum_{j=1}^{N}|[\epsilon]|_{j+1 / 2}^{2} \leq C|\epsilon|_{\Gamma}^{2} \leq C h^{2 k+1} .
$$

To estimate the first two sums in $H$, we use the following Taylor expansions

$$
\begin{gathered}
f(U)-f(u)=f^{\prime}(U)(e-\epsilon)-\frac{f^{\prime \prime}}{2}(e-\epsilon)^{2}, \\
f(U)-f(\{u\})=f^{\prime}(U)(\{e\}-\{\epsilon\})-\frac{\tilde{f}^{\prime \prime}}{2}(\{e\}-\{\epsilon\})^{2},
\end{gathered}
$$

where $f^{\prime \prime}$ and $\tilde{f}^{\prime \prime}$ are the mean values. These imply the following regrouping

$$
H_{1}:=H-H_{2}=H_{11}+H_{12}+H_{13},
$$

where

$$
\begin{aligned}
& H_{11}=\sum_{j=1}^{N} \int_{I_{j}} f^{\prime}(U) e \partial_{x} e d x+\sum_{j=1}^{N}\left(f^{\prime}(U)\{e\}[e]\right)_{j+\frac{1}{2}}, \\
& H_{12}=-\left(\sum_{j=1}^{N} \int_{I_{j}} f^{\prime}(U) \epsilon \partial_{x} e d x+\sum_{j=1}^{N}\left(f^{\prime}(U)\{\epsilon\}[e]\right)_{j+\frac{1}{2}}\right), \\
& H_{13}=-\frac{1}{2}\left(\sum_{j=1}^{N} \int_{I_{j}} f^{\prime \prime}(e-\epsilon)^{2} \partial_{x} e d x+\sum_{j=1}^{N}\left(\tilde{f}^{\prime \prime}(\{e\}-\{\epsilon\})^{2}[e]\right)_{j+\frac{1}{2}}\right)
\end{aligned}
$$

will be estimated separately as below. 
For the $H_{11}$ term, a simple integration by parts gives

$$
H_{11}=-\frac{1}{2} \sum_{j=1}^{N} \int_{I_{j}} f^{\prime \prime}(U) \partial_{x} U e^{2} d x \leq C\|e\|^{2} .
$$

Using Young's inequality and (30) we obtain

$$
\begin{aligned}
H_{12} \leq & \frac{\gamma}{3} \sum_{j=1}^{N} \int_{I_{j}} e_{x}^{2} d x+\frac{3}{4 \gamma} \sum_{j=1}^{N} \int_{I_{j}}\left(f^{\prime}(U) \epsilon\right)^{2} d x \\
& +\frac{\gamma}{3} \sum_{j=1}^{N} \beta_{0} \frac{[e]^{2}}{h}+\frac{3 h}{4 \beta_{0} \gamma} \sum_{j=1}^{N}\left(f^{\prime}(U)\{\epsilon\}\right)_{j+\frac{1}{2}}^{2} \\
\leq & \frac{\gamma}{3}\|e\|_{E}^{2}+C h^{2 k+2} .
\end{aligned}
$$

To deal with the higher order terms we use both projection and inverse inequalities, (30) and (36), to get

$$
\begin{aligned}
H_{13} \leq & \frac{\gamma}{3} \sum_{j=1}^{N} \int_{I_{j}}\left(\partial_{x} e\right)^{2} d x+\frac{3}{16 \gamma} \sum_{j=1}^{N} \int_{I_{j}}\left(f^{\prime \prime}(e-\epsilon)^{2}\right)^{2} d x \\
& +\frac{\gamma}{3} \sum_{j=1}^{N} \beta_{0} \frac{[e]^{2}}{h}+\frac{3 h}{16 \beta_{0} \gamma} \sum_{j=1}^{N}\left(\tilde{f}^{\prime \prime}\{e-\epsilon\}^{2}\right)_{j+\frac{1}{2}}^{2} \\
\leq & \frac{\gamma}{3}\|e\|_{E}^{2}+C\|e-\epsilon\|_{\infty}^{2}\left(\|e\|^{2}+\|\epsilon\|^{2}+h\|\epsilon\|_{\Gamma_{h}}^{2}\right. \\
& \left.+h\|\epsilon\|_{\Gamma_{h}}^{2}+h\|e\|_{\Gamma_{h}}^{2}\right) .
\end{aligned}
$$

Plugging all above estimates on $H$ into the inequality (154), we obtain

$$
\frac{d}{d t}\|e\|^{2} \leq C\left(\|e\|^{2}+h^{2 k+2}\right)\left(1+\|e-\epsilon\|_{\infty}^{2}\right) .
$$

Using the approximation results in (30) and (36), we have

$$
\begin{aligned}
\frac{d}{d t}\|e\|^{2} & \leq C\left(\|e\|^{2}+h^{2 k+2}\right)\left(1+h^{-1}\|e\|^{2}+h^{2 k+1}\right) \\
& \leq C\left[\|e\|^{2}\left(1+h^{-1}\|e\|^{2}\right)+h^{2 k+2}\right] .
\end{aligned}
$$

Note that for $u_{0}$ to be the standard $L^{2}$ projection of $U_{0}$ the initial error gives

$$
\left\|e_{0}\right\|=\left\|U_{0}-u^{0}\right\| \leq C h^{k+1} \text {. }
$$

For fixed finite time interval $[0, T]$, integration of (60) over $[0, t]$ for $t \leq T$ gives

$$
\|e(t)\|^{2} \leq C(T)\left[h^{2 k+2}+\int_{0}^{t}\|e(\tau)\|^{2}\left(1+h^{-1}\|e(\tau)\|^{2}\right) d \tau\right]:=C W .
$$

This implies that $W(0)=h^{2 k+2}$, and

$$
\frac{d}{d t} W=\|e(t)\|^{2}\left(1+h^{-1}\|e(t)\|^{2}\right) \leq C W\left(1+C h^{-1} W\right) .
$$

We define a constant $\widetilde{C}$ depending on $T$ such that

$$
C T=\int_{1}^{\widetilde{C}} \frac{d \eta}{\eta\left(1+C \eta h^{2 k+1}\right)}=: G(\widetilde{C}),
$$


which ensures a uniformly bound $\widetilde{C}$ in terms of $h$. Integration of inequality (61) leads to

$$
G(W / W(0)) \leq C T=G(\widetilde{C}) .
$$

Hence $W(T) \leq \widetilde{C} W(0)=\widetilde{C} h^{2 k+2}$, leading to $\|e\|^{2} \leq C W \leq C h^{2 k+2}$, implying the desired error estimate.

\section{Extension to MULTi-Dimensions}

The extension to multi-dimensions with rectangular meshes is straightforward, for which we need to first clarify some notations. Consider the following model equation

$$
\partial_{t} U+\nabla_{x} \cdot f(U)=\Delta_{x} U, \quad t>0,
$$

posed on $x=\left(x^{1}, \ldots, x^{d}\right) \in \Omega=\Pi_{i=1}^{d}\left[0, L_{x^{i}}\right] \subset \mathbb{R}^{d}$ with periodic boundary conditions. Here $f: \mathbb{R} \rightarrow \mathbb{R}^{d}$ is a smooth vector function.

6.1. Scheme formulation. For a rectangular partition of

$$
\Omega=\bigcup_{\alpha=1}^{N} K_{\alpha},
$$

where $\alpha=\left(\alpha_{1}, \ldots \alpha_{d}\right), N=\left(N_{1}, \ldots, N_{d}\right)$. Here we use rectangular meshes $\{K\} \subset$ $T_{h}$, with $K_{\alpha}=I_{\alpha_{1}}^{1} \times \cdots \times I_{\alpha_{d}}^{d}$, where $I_{\alpha_{i}}^{i}=\left[x_{\alpha_{i}-1 / 2}^{i}, x_{\alpha_{i}+1 / 2}^{i}\right]$ for $\alpha_{i}=1, \ldots, N_{i}$. The cell lengths are denoted by $h^{x^{i}}=\max _{1 \leq \alpha_{i} \leq N_{i}}\left|I_{\alpha_{i}}^{i}\right|$, with $h=\max _{1 \leq i \leq d} h^{x^{i}}$ being the maximum mesh size. We again assume that the mesh is regular.

We define the DG space as the space of tensor product of piecewise polynomials of degree at most $k$ in each variable on every element, i.e.,

$$
\mathbb{W}_{h}^{k}=\left\{v: \quad v \in Q^{k}\left(K_{\alpha}\right), \forall x \in K_{\alpha}, \alpha=1, \ldots, N\right\},
$$

where $Q^{k}$ is the space of tensor products of one-dimensional polynomials of degree up to $k$. For the one-dimensional case, we have $Q^{k}(K)=P^{k}(K)$, which is the space of polynomials of degree at most $k$ defined on $K$.

We also introduce some trace operators that will help us to define the interface terms. Let $K^{1}$ and $K^{2}$ be two neighboring cells with a common edge $e$; for $w$ defined on $\partial K^{i}, i=1,2$, we define the average $\{w\}$ and the jump $[w]$ as

$$
\{w\}=\frac{1}{2}\left(w_{1}+w_{2}\right), \quad[w]=w_{2}-w_{1} \quad \text { on } \quad e,
$$

where the jump is calculated as a forward difference along the normal direction $\vec{n}$, which is defined to be oriented from $K^{1}$ to $K^{2}$, with $w_{i}=\left.w\right|_{\partial K^{i}}$. We start with the weak formulation of (62) of the form

$$
\int_{\Omega} \partial_{t} U v d x+\int_{\Omega} \nabla U \cdot \nabla v d x=\int_{\Omega} f(U) \cdot \nabla_{x} v d x, \forall v \in H_{0}^{1}(\Omega),
$$

and approximate $U$ by $u \in \mathbb{W}_{h}^{k}$, and also allow $v \in \mathbb{W}_{h}^{k}$ to be discontinuous crossing $\partial K$. With such a DG discretization the above formulation has to be refined by including some interface corrections, leading to the following DG scheme,

$$
\left\langle u_{t}, v\right\rangle+A(u, v)=F(u, v),
$$


where $\langle\cdot, \cdot\rangle$ denotes the $L^{2}$ inner product over $\Omega$, and

$$
\begin{aligned}
& A(u, v)=\sum_{K \in T_{h}} \int_{K} \nabla_{x} u \cdot \nabla_{x} v d x+\sum_{e \in \Gamma} \int_{e}\left(\widehat{\partial_{n} u}[v]+[u]\left\{\partial_{n} v\right\}\right) d s, \\
& F(u, v)=\sum_{K \in T_{h}} \int_{K} f(u) \cdot \nabla_{x} v d x+\sum_{e \in \Gamma} \int_{e} \widehat{f^{n}}[v] d s .
\end{aligned}
$$

This is a complete DG discretization as long as the "hat" terms are defined.

6.2. Stability. For nonlinear flux we take $\hat{f}$ as an entropy satisfying numerical flux: let $e=\partial K^{1} \cap \partial K^{2}$ and $u_{i}=\left.u\right|_{\partial K^{i}}$,

$$
\int_{e}\left(\widehat{f^{n}}\left(u_{1}, u_{2}\right)-f(\xi) \cdot \vec{n}\right)[u] d s \leq 0
$$

for all $\xi$ between $u_{1}$ and $u_{2}$. For the directional derivative of the solution induced from the diffusion we take

$$
\widehat{\partial_{n} u}=\beta_{0} h^{-1}[u]+\left\{\partial_{n} u\right\}+\beta_{1} h\left[\partial_{n}^{2} u\right]
$$

with $\left(\beta_{0}, \beta_{1}\right)$ chosen so that $\beta_{0}$ is suitably large $\left(>\Gamma\left(\beta_{1}\right)\right)$ to ensure the following coercivity

$$
A(v, v) \geq \gamma\|v\|_{E}^{2}
$$

for some $\gamma>0$, where

$$
\|v\|_{E}^{2}=\sum_{K \in T_{h}} \int_{K}\left|\nabla_{x} v\right|^{2} d x+\sum_{e \in \Gamma} \int_{e} \frac{\beta_{0}}{h}[v]^{2} d s, v \in \mathbb{W}_{h}^{k} .
$$

Lemma 6.1. For the entropy flux $\hat{f}$ defined in (65), it holds that

$$
F(v, v) \leq 0, \quad \forall v \in \mathbb{W}_{h}^{k} .
$$

Proof. Set $g_{i}^{\prime}(v)=f_{i}(v)$ with $g(v)=\left(g_{1}(v), \ldots, g_{d}(v)\right)$, then

$$
\int_{K} f(v) \cdot \nabla_{x} v d x=\int_{K} \nabla_{x} \cdot g(v) d x=\int_{\partial K} g(v) \cdot \vec{n}_{K} d s,
$$

which can be rewritten as

$$
\int_{\partial K}\{g(v)\} \cdot \vec{n}_{K} d s-\frac{1}{2} \int_{\partial K}[g(v)] \cdot \vec{n}_{K} d s .
$$

Hence, using (65) we have

$$
\begin{aligned}
F(v, v) & =\sum_{e \in \Gamma}\left[-\int_{e}[g] \cdot \vec{n} d s+\int_{e} \widehat{f^{n}}[v] d s\right] \\
& =\sum_{e \in \Gamma} \int_{e}\left(\widehat{f^{n}}-f(\xi) \cdot \vec{n}\right)[v] d s \leq 0,
\end{aligned}
$$

where we have used the mean value theorem and the definition of $g$; $\xi$ is between $\left.v\right|_{\partial K}$ and $v^{\text {ext }}$.

Both $A(u, u) \geq \gamma\|u\|_{E}^{2}$ and $F(u, u) \leq 0$ imply the desired energy stability

$$
\frac{d}{d t}\|u\|^{2} \leq-\gamma\|u\|_{E}^{2} \leq 0 \text {. }
$$


6.3. Projection and projection properties. To prove the error estimates for multi-dimensional problems with rectangular meshes, we need a suitable projection similar to the one-dimensional case. Such a projection can be defined as

$$
\Pi w=P^{\left(x^{1}\right)} \otimes \cdots \otimes P^{\left(x^{d}\right)} w,
$$

where the superscripts indicate the application of the one-dimensional operator $P^{\left(x^{i}\right)}$ with respect to the corresponding variable $x^{i}$.

In other words, for a given (piecewise) smooth function $w$, the projection $\Pi w$ is the unique function in $\mathbb{W}_{h}^{k}$ defined in (69), with $P^{\left(x^{i}\right)}$ determined by

$$
\begin{aligned}
& \int_{I_{\alpha_{i}}^{i}}\left(P^{\left(x^{i}\right)} w(x)-w(x)\right) \partial_{x^{i}}^{2} v(x) d x^{i}=0, \quad \forall v \in P^{k}\left(I_{\alpha_{i}}^{i}\right), \alpha_{i}=1, \ldots, N_{i}, \\
& \left.\partial_{x^{i}} \widehat{\left(P^{\left(x^{i}\right)}\right.} w\right):=\beta_{0}\left(h^{i}\right)^{-1}\left[P^{\left(x^{i}\right)} w\right] \\
& \quad+\left\{\partial_{x^{i}}\left(P^{\left(x^{i}\right)} w\right)\right\}+\left.\beta_{1} h^{i}\left[\partial_{x^{i}}^{2}\left(P^{\left(x^{i}\right)} w\right)\right]\right|_{x_{\alpha_{i}+1 / 2}^{i}}=\left.\widehat{\partial_{x^{i}} w}\right|_{x_{\alpha_{i}+1 / 2}^{i}},
\end{aligned}
$$

$$
\left.\left\{P^{\left(x^{i}\right)} w\right\}\right|_{x_{\alpha_{i}+1 / 2}^{i}}=\left.\{w\}\right|_{x_{\alpha_{i}+1 / 2}^{i}},
$$

where periodic extensions are used at the domain boundary. Similar to the onedimensional case, there are some approximation results for the above multidimensional projection:

$$
\|\epsilon\|+h^{\frac{d+1}{2}}\|\epsilon\|_{\infty}+h^{1 / 2}\|\epsilon\|_{\Gamma_{h}} \leq C h^{k+1},
$$

where $\epsilon=\Pi U-U$. The positive constant $C$, solely depending on $|U|_{k+1}$, is independent of $h . \Gamma_{h}$ denotes the boundary sets of all elements $K_{\alpha}$. We present more refined estimates than (71) in Theorem 7.3. The proof presented in $\S 7$ for the two-dimensional setting is for simplicity only.

Finally, we list some inverse properties of the finite element space $\mathbb{W}_{h}^{k}$ that will be used in our error analysis. For any function $w_{h} \in \mathbb{W}_{h}^{k}$, the following inverse inequalities hold [13]:

$$
\begin{aligned}
&\left\|\partial_{x}^{l} w_{h}\right\| \leq C h^{-l}\left\|w_{h}\right\|, \\
&\left\|w_{h}\right\|_{\Gamma_{h}} \leq C h^{-1 / 2}\left\|w_{h}\right\|, \\
&\left\|w_{h}\right\|_{\infty} \leq C h^{-d / 2}\left\|w_{h}\right\|,
\end{aligned}
$$

where $d$ is the spatial dimension. For more details of these inverse properties, we refer to 13 .

6.4. Error estimates. The stability result also yields the following error estimate.

Theorem 6.2. Let $u$ be the solution to the semidiscrete DDG scheme (63) for problem (62) subject to initial data $U_{0}$ using polynomial elements of degree $k$, and $U$ be a smooth solution of (62) subject to initial $U_{0}$. If $k \geq \frac{d-1}{2}$, then the following error estimate holds:

$$
\int_{\Omega}(u(x, t)-U(x, t))^{2} d x \leq C h^{2(k+1)},
$$

where $C$ depends solely on $|U|_{k+2}$ and data given, but is independent of $h$. 
Remark 6.1. Note that for purely diffusion problem with no convection (i.e., $f(u)=$ 0 ) or with linear diffusion $f(u)=c u$, the restriction $k \geq \frac{d-1}{2}$ is unnecessary. For nonlinear convection, this restriction suggests that one may use polynomials of degree $k \geq 1$ for $d=2,3$.

Remark 6.2. In the multi-dimensional case, the proof requires a stronger smoothness assumptions on the exact solution than the ones in the one-dimensional case. In this sense, Theorem 6.2 may be regarded as a superconvergence result.

Proof. Let $\widetilde{U} \in \mathbb{W}_{h}^{k}$ be determined to approximate $U$, and set

$$
w=\widetilde{U}-u, \quad \epsilon=\widetilde{U}-U
$$

so that $U-u=w-\epsilon$, We take $v=w$ in (63) to obtain

$$
\left\langle\partial_{t} w, w\right\rangle+A(w, w)=\left\langle\partial_{t} \epsilon, w\right\rangle+A(\epsilon, w)+H, \quad H:=F(U, w)-F(u, w) .
$$

The choice of $\left(\beta_{0}, \beta_{1}\right)$ can be made so that

$$
A(w, w) \geq \gamma\|w\|_{E}^{2} .
$$

We take $\widetilde{U}=\Pi U$ as the projection of $U$ defined in (70). In multi-dimensional case, $A(\epsilon, w)$ is no longer zero, but still controllable with the following superconvergence result.

Lemma 6.3. Let $A$ be defined in $(64)$. Then we have for any $s \geq 0$,

$$
A(\epsilon, w) \leq C\left(\|w\|^{2}+h^{2 \min \{k, s\}+2}\right)+\frac{1}{2} A(w, w) .
$$

The constant $C$ depends on $|U|_{s+2}$.

We postpone the proof of this result to the end of this section, and proceed to estimate $H$ in (75). We take the average of $\left.u\right|_{\partial K^{i}}$ as a reference value $\{u\}$, hence

$$
\begin{aligned}
H= & \sum_{K \in T_{h}} \int_{K}(f(U)-f(u)) \cdot \nabla_{x} w d x+\int_{\Gamma}\left(f^{n}(U)-f^{n}(\{u\})\right)[w] d s \\
& +\int_{\Gamma}\left(f^{n}(\{u\})-\widehat{f}^{n}\right)[w] d s
\end{aligned}
$$

where we use the notation $f^{n}(\xi)=f(\xi) \cdot \vec{n}$ and have taken the periodic boundary condition into account.

Using the Taylor expansions

$$
\begin{gathered}
f(U)-f(u)=f^{\prime}(U)(w-\epsilon)-\frac{f^{\prime \prime}}{2}(w-\epsilon)^{2}, \\
f(U)-f(\{u\})=f^{\prime}(U)(\{w\}-\{\epsilon\})-\frac{\tilde{f}^{\prime \prime}}{2}(\{w\}-\{\epsilon\})^{2},
\end{gathered}
$$

where $f^{\prime \prime}$ and $\tilde{f}^{\prime \prime}$ are the mean values, and $\alpha^{n}(\hat{f} ; \xi):=[u]^{-1}(f(\xi)-\hat{f})$ with $[u]=$ $[\epsilon]-[w]$ we obtain

$$
H=H_{1}+H_{2}+H_{3}+H_{4}
$$


where

$$
\begin{aligned}
& H_{1}=\sum_{K \in T_{h}} \int_{K} w f^{\prime}(U) \cdot \nabla_{x} w d x+\int_{\Gamma} \vec{n} \cdot f^{\prime}(U)\{w\}[w] d s \\
& H_{2}=-\left(\sum_{K \in T_{h}} \int_{K} \epsilon f^{\prime}(U) \cdot \nabla_{x} w d x+\int_{\Gamma} \vec{n} \cdot f^{\prime}(U)\{\epsilon\}[w] d s\right), \\
& H_{3}=-\frac{1}{2}\left(\sum_{K \in T_{h}} \int_{K}(w-\epsilon)^{2} f^{\prime \prime} \cdot \nabla_{x} w d x+\int_{\Gamma} \vec{n} \cdot \tilde{f}^{\prime \prime}(\{w\}-\{\epsilon\})^{2}[w] d s\right), \\
& H_{4}=-\int_{\Gamma} \alpha^{n}(\hat{f} ;\{u\})[w]^{2} d s+\int_{\Gamma} \alpha^{n}(\hat{f} ;\{u\})[\epsilon][w] d s
\end{aligned}
$$

will be estimated separately as below.

For the $H_{1}$ term, a simple integration by parts gives

$$
\begin{aligned}
H_{1} & =\int_{\Gamma} \vec{n} \cdot f^{\prime}(U)\{w\}[w] d s-\frac{1}{2} \int_{\Gamma} \vec{n} \cdot f^{\prime}(U)\left[w^{2}\right] d s+\frac{1}{2} \sum_{K \in T_{h}} \int_{K} f^{\prime \prime}(U) \cdot \nabla_{x} U w^{2} d x \\
& =\frac{1}{2} \sum_{K \in T_{h}} \int_{K} f^{\prime \prime}(U) \cdot \nabla_{x} U w^{2} d x \leq C\|w\|^{2} .
\end{aligned}
$$

Using Young's inequality and (171) we obtain

$$
\begin{aligned}
H_{2} \leq & \frac{\gamma}{6} \sum_{K \in T_{h}} \int_{K}\left|\nabla_{x} w\right|^{2} d x+\frac{3}{2 \gamma} \sum_{K \in T_{h}} \int_{K}\left(f^{\prime}(U) \epsilon\right)^{2} d x \\
& +\frac{\gamma}{6} \int_{\Gamma} \beta_{0} \frac{[w]^{2}}{h} d s+\frac{3 h}{2 \beta_{0} \gamma} \int_{\Gamma}\left(f^{\prime}(U) \epsilon\right)^{2} d s \\
\leq & \frac{\gamma}{6}\|w\|_{E}^{2}+C h^{2 k+2} .
\end{aligned}
$$

To deal with the higher order terms we use both projection and inverse inequalities, (71) and (72), to get

$$
\begin{aligned}
H_{3} \leq & \frac{\gamma}{6} \sum_{K \in T_{h}} \int_{K}\left|\nabla_{x} w\right|^{2} d x+\frac{3}{8 \gamma} \sum_{K \in T_{h}} \int_{K}\left(f^{\prime \prime}(w-\epsilon)^{2}\right)^{2} d x \\
& +\frac{\gamma}{6} \int_{\Gamma} \beta_{0} \frac{[w]^{2}}{h} d s+\frac{3 h}{8 \beta_{0} \gamma} \int_{\Gamma}\left(\tilde{f}^{\prime \prime}\{w-\epsilon\}^{2}\right)^{2} d s \\
\leq & \frac{\gamma}{6}\|w\|_{E}^{2}+C\|w-\epsilon\|_{\infty}^{2}\left(\|w\|^{2}+\|\epsilon\|^{2}\right) .
\end{aligned}
$$

For the entropy flux, the first term in $H_{4}$ is nonpositive; using the Young inequality, we bound the last term further by

$$
\begin{aligned}
H_{4} & \leq \frac{\gamma}{6} \int_{\Gamma} \beta_{0} \frac{[w]^{2}}{h} d s+\frac{3 h}{2 \beta_{0} \gamma} \int_{\Gamma}\left(\left(\alpha^{n}\right)^{2}[\epsilon]^{2}\right) d s \\
& \leq \frac{\gamma}{6} \int_{\Gamma} \beta_{0} \frac{[w]^{2}}{h} d s+C h^{2 k+2} .
\end{aligned}
$$

The above estimates lead to

$$
H \leq \frac{\gamma}{2}\|w\|_{E}^{2}+C\left(\|w\|^{2}+h^{2 k+2}\right)\left(1+\|w-\epsilon\|_{\infty}^{2}\right) .
$$


Substituting this, (76) and (77) into the equality (75), we obtain

$$
\frac{d}{d t}\|w\|^{2} \leq C\left(\|w\|^{2}+h^{2 k+2}\right)\left(1+\|w-\epsilon\|_{\infty}^{2}\right) .
$$

Using the approximation results (171) and (172), for small $h$, we have

$$
\|w-\epsilon\|_{\infty} \leq\|w\|_{\infty}+\|\epsilon\|_{\infty} \leq C\left(h^{-d / 2}\|w\|+h^{k+1-\frac{d+1}{2}}\right) \leq C\left(h^{-d / 2}\|w\|+1\right),
$$

where we have used the assumption that $k \geq \frac{d-1}{2}$. Hence (81) reduces to

$$
\frac{d}{d t}\|w\|^{2} \leq C\left[\|w\|^{2}\left(1+h^{-d}\|w\|^{2}\right)+h^{2 k+2}\right] .
$$

Using the same argument as that estimating (60), with $G$ replaced by

$$
C T=G(\widetilde{C})=\int_{1}^{\widetilde{C}} \frac{d \eta}{\eta\left(1+C \eta h^{2 k+2-d}\right)},
$$

which defines a uniform bound of $\widetilde{C}$ in terms of $h$ since $2 k+2-d \geq 1$, we obtain that $\|w\|^{2} \leq C \widetilde{C} h^{2 k+2}$, leading to the desired error estimate.

Proof of Lemma 6.3. Let us use the notation $\int_{\Gamma}:=\sum_{e \in \Gamma} \int_{e}$. A straightforward calculation shows that for all $K \in T_{h}$,

$$
\sum_{K \in T_{h}} \int_{\partial K} \epsilon \partial_{n} w d s=-\int_{\Gamma}[\epsilon] \cdot\left\{\partial_{n} w\right\} d s-\int_{\Gamma}\{\epsilon\} \cdot\left[\partial_{n} w\right] d s
$$

with which we have

$$
\begin{aligned}
A(\epsilon, w) & =\sum_{K \in T_{h}} \int_{K} \nabla_{x} \epsilon \cdot \nabla_{x} w d x+\int_{\Gamma}\left(\widehat{\partial_{n} \epsilon}[w]+[\epsilon]\left\{\partial_{n} w\right\}\right) d s \\
& =-\sum_{K \in T_{h}} \int_{K} \Delta_{x} w \epsilon d x+\int_{\Gamma}\left(\widehat{\partial_{n} \epsilon}[w]-\{\epsilon\}\left[\partial_{n} w\right]\right) d s .
\end{aligned}
$$

In multi-dimensional case, this is no longer zero. To estimate it, we rewrite

$$
A(\epsilon, w)=\sum_{i=1}^{d} A_{i}(U, w)
$$

where

$$
A_{i}(U, w)=-\sum_{\alpha=1}^{N} \int_{K_{\alpha}} w_{x^{i} x^{i}} \epsilon d x+\int_{K_{\alpha} / I_{\alpha_{i}}^{i}}\left(\widehat{\partial_{x^{i}}} \epsilon[w]-\{\epsilon\}\left[\partial_{x^{i}} w\right]\right)_{x_{\alpha_{i}+1 / 2}^{i}} d \hat{x}^{i}
$$

with $\epsilon=\Pi U-U$ and $d \hat{x}^{i}=\prod_{j \neq i} d x^{j}$. The proof of the approximation results for $A_{i}, i=1 \cdots d$ is analogous; therefore we only present the one for $A_{1}$. Here we use an argument similar to the one in the proof of Lemma 3.6 in [9] for a local projection, with the unsettled part further absorbed by $A(w, w)$.

We first claim that

$$
A_{1}(U, w)=0,\left.\quad \forall U\right|_{K_{\alpha}} \in P^{k+1}\left(K_{\alpha}\right), w \in \mathbb{W}_{h}^{k} .
$$

To prove this claim, we fix $w \in \mathbb{W}_{h}^{k}$. Since $\Pi$ is a polynomial preserving operator, (83) holds true for every $U \in \mathbb{W}_{h}^{k}$. Therefore, we only need to consider the cases

$$
\left.U(x)\right|_{K_{\alpha}}=a_{\alpha}\left(x^{j}\right)^{k+1}
$$

where constant $a_{\alpha}$ may vary from element to element. Below we shall use the notation $U_{i}=U\left(x^{i}\right)$ to denote the dependence only on variable $x^{i}$. 
For $j=1$, we have $\Pi U=P^{\left(x^{1}\right)} U_{1}$ and $w_{x^{1} x^{1}}$ is a polynomial of degree at most $k-2$ in $x^{1}$, we obtain

$$
\sum \int_{K_{\alpha}}(\Pi U-U) w_{x^{1} x^{1}} d x=\sum \int_{K_{\alpha}}\left(P^{\left(x^{1}\right)} U_{1}-U_{1}\right) w_{x^{1} x^{1}} d x=0
$$

In addition, we have

$$
\left.\{\Pi U\}=\left\{P^{\left(x^{1}\right)} U_{1}\right\}=\{U\}, \quad \widehat{\partial_{x^{1}}(\Pi U}\right)=\partial_{x^{1}} \widehat{P^{\left(x^{1}\right)}} U_{1}=\widehat{\partial_{x^{1}} U}
$$

Thus, $A_{1}(U, w)=0$ for $\left.U\right|_{K_{\alpha}}=a_{\alpha}\left(x^{1}\right)^{k+1}$.

In the case $j \neq 1$, we integrate by parts and obtain

$$
A_{1}(U, w)=-\sum \int_{K_{\alpha} / I_{1}^{x^{1}}} d \hat{x}^{1}\left(-\int_{I_{1}^{x^{1}}} w_{x^{1}} \partial_{x^{1}} \epsilon d x^{1}+\left(\widehat{\partial_{x^{1}}} \epsilon[w]+\left\{w_{x^{1}}\right\}[\epsilon]\right)_{x_{\alpha_{1}+1 / 2}^{1}}\right) .
$$

Due to the special form of $U$ we have $\partial_{x^{1}} \epsilon=\partial_{x^{1}}(\Pi U-U)=0$ and

$$
\Pi U=P^{\left(x^{j}\right)} U_{j}
$$

on the interface $x^{1}=x_{\alpha_{1}+1 / 2}^{1}$, where $\widehat{\partial_{x^{1}} \epsilon}=0$ and $[\epsilon]=0$ by a direct check. We thus conclude that $A_{1}(U, w)=0$ for $\left.U\right|_{K_{\alpha}}=a_{\alpha}\left(x^{j}\right)^{k+1}$. This completes the proof of 83 .

For fixed $w \in \mathbb{W}_{h}^{k}$, the linear functional $U \rightarrow A_{1}(U, w)$ is continuous on $H^{s+2}$ with norm bounded by $C\left[\sum_{\alpha}\|w\|_{2, K_{\alpha}}^{2}\right]^{1 / 2}$. Due to (3), it vanishes over $P^{s+1}$ for any $0 \leq s \leq k$. Hence, by applying the Bramble-Hilbert lemma combined with the standard scaling argument, we obtain for $U \in H^{s+2}$ that

$$
\left|A_{1}(U, w)\right| \leq C h^{s+2}|U|_{s+2}\left[\sum_{\alpha}\|w\|_{2, K_{\alpha}}^{2}\right]^{1 / 2} .
$$

Applying this to all $A_{i}$ and using the inverse inequality $(72$ a), we proceed with

$$
\begin{aligned}
|A(U, w)| & \leq C h^{s+2}|U|_{s+2}\left(\|w\|^{2}+\left(1+C h^{-2}\right) \sum_{\alpha}\left\|\nabla_{x} w\right\|_{0, K_{\alpha}}^{2}\right)^{1 / 2} \\
& \leq C h^{s+2}|U|_{s+2}\left(\|w\|^{2}+h^{-2}\|w\|_{E}^{2}\right)^{1 / 2} \\
& \leq C h^{s+2}|U|_{s+2}\left(\|w\|+h^{-1}\|w\|_{E}\right) \\
& \leq C h^{s+2}|U|_{s+2}\|w\|+\frac{C^{2}}{2 \gamma}|U|_{s+2}^{2} h^{2 s+2}+\frac{\gamma}{2}\|w\|_{E}^{2} \\
& \leq C\left(|U|_{s+2}\right)\left(\|w\|^{2}+h^{2 s+2}\right)+\frac{1}{2} A(w, w) .
\end{aligned}
$$

Hence we have proved Lemma 6.3 


\section{SOME ESTIMATES FOR THE PROJECTION ERROR}

Theorem 7.1 (The projection estimates). Let $P: H^{s+1} \rightarrow \mathbb{V}_{h}^{k}$ denote the onedimensional global projection (29). If $\left.w\right|_{I_{j}} \in H^{s+1}\left(I_{j}\right)$ for $j=1, \ldots, N$, then we have the following estimates:

$$
\begin{gathered}
\sum_{j=1}^{N}\|P w-w\|_{0, I_{j}}^{2} \leq C h^{2 p+2} \sum_{j=1}^{N}|w|_{p+1, I_{j}}^{2}, \\
\sum_{j=1}^{N}|P w-w|_{j+1 / 2}^{2} \leq C h^{2 p+1} \sum_{j=1}^{N}|w|_{p+1, I_{j}}^{2}, \\
\sum_{j=1}^{N}|P w-w|_{\infty, I_{j}}^{2} \leq C h^{2 p} \sum_{j=1}^{N}|w|_{p+1, I_{j}}^{2},
\end{gathered}
$$

for any $1 \leq p \leq \min \{k, s\}$, where $C$ depends on $k, \beta_{0}, \beta_{1}$ but is independent of $I_{j}$ and $w$.

This leads to the estimates stated in Lemma 4.2,

We present a self-contained proof using Legendre polynomials; see 34 for details of using Legendre polynomials to estimate the local projection error.

Proof. This proof is carried out in four steps.

Step 1. First, we derive bounds on the difference $w-P w$ in terms of the Legendre coefficients of $w$. To do so, denote by $\phi_{i+1}=L_{i}(\xi), i \geq 0$, the Legendre polynomial of degree $i$ on $I=[-1,1]$ and expand the function $w$ and $P w$ on $I_{j}$ into the series

$$
\begin{aligned}
\left.w\right|_{I_{j}}=\widetilde{w}_{j}(\xi) & :=\sum_{i=1}^{\infty} \omega_{i}^{j} \phi_{i}(\xi), \\
\left.P w\right|_{I_{j}}=\widetilde{P w_{j}}(\xi) & :=\sum_{i=1}^{k-1} \omega_{i}^{j} \phi_{i}(\xi)+a_{k}^{j} \phi_{k}(\xi)+a_{k+1}^{j} \phi_{k+1}(\xi),
\end{aligned}
$$

which satisfies the orthogonal property $(29 \mathrm{a})$. Hence we have

$$
\left.(w-P w)\right|_{I_{j}}=\sum_{i=k+2}^{\infty} \omega_{i}^{j} \phi_{i}(\xi)+\left(\omega_{k}^{j}-a_{k}^{j}\right) \phi_{k}(\xi)+\left(\omega_{k+1}^{j}-a_{k+1}^{j}\right) \phi_{k+1}(\xi) .
$$

Let $Q_{k}$ be the standard $L^{2}$-projection from $L^{2}(I)$ onto $P^{k}(I)$ with

$$
Q_{k} \widetilde{w}_{j}=\sum_{i=1}^{k+1} \omega_{i}^{j} \phi_{i}(\xi)
$$

Then

$$
\inf _{v \in P^{k}[-1,1]}\left\|\widetilde{w}_{j}-v\right\|=\left\|\widetilde{w}_{j}-Q_{k} \widetilde{w}_{j}\right\|
$$


where $\widetilde{w}_{j}-Q_{k} \widetilde{w}_{j}=\sum_{i=k+2}^{\infty} \omega_{i}^{j} \phi_{i}(\xi)$ with $\left\|\phi_{i}\right\|^{2}=2 /(2 i-1)$ and $\phi_{i}( \pm 1)=( \pm 1)^{i+1}$, we obtain from the above expression the following bounds:

$$
\begin{aligned}
\left\|\widetilde{w}_{j}-{\widetilde{P w_{j}}}\right\|^{2} & =\left\|\widetilde{w}_{j}-Q_{k} \widetilde{w}_{j}\right\|^{2}+\sum_{i=k}^{k+1}\left|\omega_{i}^{j}-a_{i}^{j}\right|^{2} \frac{2}{2 i-1}, \\
\left(\widetilde{w}_{j}-{\widetilde{P w_{j}}}\right)(+1) & =\sum_{i=k+2}^{\infty} \omega_{i}^{j}+\sum_{i=k}^{k+1}\left(\omega_{i}^{j}-a_{i}^{j}\right), \\
\left|\left(\widetilde{w}_{j}-{\widetilde{P w_{j}}}\right)(\xi)\right| & \leq\left|\widetilde{w}_{j}-Q_{k} \widetilde{w}_{j}\right|_{\infty}+\sum_{i=k}^{k+1}\left|\omega_{i}^{j}-a_{i}^{j}\right| \cdot\left|\phi_{i}\right|_{\infty} .
\end{aligned}
$$

Step 2. In order to estimate the sum of the coefficient $\omega_{i}^{j}$, following [11, we consider

$$
\partial_{\xi} \widetilde{w}_{j}(\xi)=\sum_{i=1}^{\infty} \beta_{i}^{j} \phi_{i}(\xi)
$$

Integration of this yields

$$
\widetilde{w}_{j}(\xi)=\widetilde{w}_{j}(-1)+\sum_{i=1}^{\infty} \beta_{i}^{j} \int_{-1}^{\xi} \phi_{i}(\nu) d \nu,
$$

and for $i \geq 2$ using the identity

$$
\int_{-1}^{\xi} \phi_{i}(\nu) d \nu=\frac{1}{2 i-1}\left(\phi_{i+1}(\xi)-\phi_{i-1}(\xi)\right),
$$

we obtain

$$
\widetilde{w}_{j}(\xi)=\left(\widetilde{w}_{j}(-1)+\beta_{1}^{j}\right) \phi_{1}+\sum_{i=2}^{\infty} \frac{\beta_{i-1}^{j}}{2 i-3} \phi_{i}(\xi)-\sum_{i=1}^{\infty} \frac{\beta_{i+1}^{j}}{2 i+1} \phi_{i}(\xi) .
$$

Comparing coefficients in the Legendre expressions, one can conclude that

$$
\omega_{i}^{j}=\frac{\beta_{i-1}^{j}}{2 i-3}-\frac{\beta_{i+1}^{j}}{2 i+1}, \quad i \geq 2
$$

therefore

$$
\begin{aligned}
\sum_{i=k+2}^{\infty} \omega_{i}^{j} & =\frac{\beta_{k+1}^{j}}{2 k+1}+\frac{\beta_{k+2}^{j}}{2 k+3}, \\
\sum_{i=k+2}^{\infty} \omega_{i}^{j}(-1)^{i+1} & =(-1)^{k+1}\left(\frac{\beta_{k+1}^{j}}{2 k+1}-\frac{\beta_{k+2}^{j}}{2 k+3}\right) .
\end{aligned}
$$

Since $\left\|\partial_{\xi} \widetilde{w}_{j}\right\|_{I}^{2}=\sum_{i=1}^{\infty}\left(\beta_{i}^{j}\right)^{2} \frac{2}{2 i-1}$, we then have

$$
\begin{gathered}
\left|\sum_{i=k+2}^{\infty} \omega_{i}^{j} \phi_{i}( \pm 1)\right|^{2} \leq \frac{1}{2 k+1}\left\|\partial_{\xi} \widetilde{w}_{j}\right\|^{2}, \\
\sum_{i=2}^{k+1}\left|\omega_{i}^{j}\right|^{2} \leq 2\left\|\partial_{\xi} \widetilde{w}_{j}\right\|^{2} .
\end{gathered}
$$


Step 3. We now estimate the difference $\omega_{i}^{j}-a_{i}^{j}$ for $i=k, k+1$. From two interface conditions (29) $-(29 \mathrm{k})$ it follows that

$$
\begin{aligned}
& \sum_{i=k}^{k+1}\left[\phi_{i}(1) a_{i}^{j}+\phi_{i}(-1) a_{i}^{j+1}\right]=2\{w\}_{j+1 / 2}-\sum_{i=1}^{k-1}\left[\phi_{i}(1) \omega_{i}^{j}+\omega_{i}^{j+1} \phi_{i}(-1)\right], \\
& \sum_{i=k}^{k+1}\left[g_{0}(i) a_{i}^{j}+g_{1}(i) a_{i}^{j+1}\right]=h \widehat{w}_{x} j+1 / 2 \\
&-\sum_{i=1}^{k-1}\left[g_{0}(i) \omega_{i}^{j}+g_{1}(i) \omega_{i}^{j+1}\right] .
\end{aligned}
$$

This can be expressed as

$$
\begin{aligned}
& \sum_{i=k}^{k+1}\left[\phi_{i}(1)\left(a_{i}^{j}-\omega_{i}^{j}\right)+\phi_{i}(-1)\left(a_{i}^{j+1}-\omega_{i}^{j+1}\right)\right]= \widetilde{b}_{1}^{j}:=2\{w\}_{j+1 / 2} \\
&-\sum_{i=1}^{k+1}\left[\phi_{i}(1) \omega_{i}^{j}+\omega_{i}^{j+1} \phi_{i}(-1)\right], \\
& \sum_{i=k}^{k+1}\left[g_{0}(i)\left(a_{i}^{j}-\omega_{i}^{j}\right)+g_{1}(i)\left(a_{i}^{j+1}-\omega_{i}^{j+1}\right)\right]=\widetilde{b}_{2}^{j}:=h \widehat{w}_{x} j+1 / 2 \\
& \\
&-\sum_{i=1}^{k+1}\left[g_{0}(i) \omega_{i}^{j}+g_{1}(i) \omega_{i}^{j+1}\right] .
\end{aligned}
$$

Since this system is uniquely solvable, using periodicity we obtain

$$
\sum_{j=1}^{N} \sum_{i=k}^{k+1}\left|a_{i}^{j}-\omega_{i}^{j}\right|^{2} \leq C \sum_{j=1}^{N}\left(\left(\widetilde{b}_{1}^{j}\right)^{2}+\left(\widetilde{b}_{2}^{j}\right)^{2}\right)
$$

From

$$
\widetilde{b}_{1}^{j}=\sum_{i=k+2}^{\infty}\left[\phi_{i}(1) \omega_{i}^{j}+\omega_{i}^{j+1} \phi_{i}(-1)\right]=\sum_{i=k+2}^{\infty} \omega_{i}^{j}+\sum_{i=k+2}^{\infty} \omega_{i}^{j+1}(-1)^{i+1}
$$

and (92) we have

$$
\sum_{j=1}^{N}\left|\widetilde{b}_{1}^{j}\right|^{2} \leq \sum_{j=1}^{N} \frac{2}{2 k+1}\left(\left\|\partial_{\xi} \widetilde{w}_{j}\right\|^{2}+\left\|\partial_{\xi} \widetilde{w}_{j+1}\right\|^{2}\right) \leq \frac{4}{2 k+1} \sum_{j=1}^{N}\left\|\partial_{\xi} \widetilde{w}_{j}\right\|^{2} .
$$

We rewrite $\widetilde{b}_{2}^{j}$ as

$$
\begin{aligned}
\widetilde{b}_{2}^{j}=\beta_{0} & \left(\widetilde{w}_{j+1}(-1)-\widetilde{w}_{j}(1)\right)-\sum_{i=1}^{k+1}\left[g_{0}(i) \omega_{i}^{j}+g_{1}(i) \omega_{i}^{j+1}\right] \\
& +\left(\partial_{\xi} \widetilde{w}_{j+1}(-1)+\partial_{\xi} \widetilde{w}_{j}(1)\right)+4 \beta_{1}\left(\partial_{\xi}^{2} \widetilde{w}_{j+1}(-1)-\partial_{\xi}^{2} \widetilde{w}_{j}(1)\right) .
\end{aligned}
$$

The terms in the first line reduce to

$$
\beta_{0} \sum_{i=k+2}^{\infty} \omega^{j+1}(-1)^{i+1}-\beta_{0} \sum_{i=k+2}^{\infty} \omega^{j}-\sum_{i=2}^{k+1}\left[\left(g_{0}(i)+\beta_{0}\right) \omega_{i}^{j}+\left(g_{1}(i)+\beta_{0}(-1)^{i}\right) \omega_{i}^{j+1}\right] .
$$

Note that here the $i=1$ term in the sum vanishes since $g_{0}(1)=-\beta_{0}$ and $g_{1}(1)=$ $(-1)^{i} g_{0}(1)$. Based on all terms in $\widetilde{b}_{2}^{j}$, using (92), (93) and $|v|_{\infty,[-1,1]}^{2} \leq 2\|v\|_{1,[-1,1]}^{2}$, 
we obtain

$$
\begin{aligned}
\sum_{j=1}^{N}\left(\widetilde{b}_{2}^{j}\right)^{2} \leq 8 \sum_{j=1}^{N}\left(\beta_{0}^{2}\left|\sum_{i=k+2}^{\infty} \omega^{j} \phi_{i}( \pm 1)\right|^{2}\right. & +k\left(\max _{i}\left|g_{0}(i)\right|+\beta_{0}\right)^{2} \sum_{i=2}^{k+1}\left(\omega_{i}^{j}\right)^{2} \\
& \left.+\left|\partial_{\xi} \widetilde{w}_{j}\right|^{2}+16 \beta_{1}^{2}\left|\partial_{\xi}^{2} \widetilde{w}_{j}\right|^{2}\right) \\
\leq 8 & \sum_{j=1}^{N}\left(\left(\frac{\beta_{0}^{2}}{2 k+1}+2 k\left(\max _{i}\left|g_{0}(i)\right|+\beta_{0}\right)^{2}\right)\left\|\partial_{\xi} \widetilde{w}_{j}\right\|^{2}\right. \\
& \left.+2\left\|\partial_{\xi} \widetilde{w}_{j}\right\|_{1}^{2}+32 \beta_{1}^{2}\left\|\partial_{\xi}^{2} \widetilde{w}_{j}\right\|_{1}^{2}\right) \\
\leq & C\left(k, \beta_{0}, \beta_{1}\right) \sum_{j=1}^{N}\left\|\partial_{\xi} \widetilde{w}_{j}\right\|_{\min \{k, 2\}}^{2} .
\end{aligned}
$$

This is valid for $k \geq 1$ since when $k=1, \beta_{1}=0$ is the default value.

These, when inserted into (94), yield

$$
\sum_{j=1}^{N} \sum_{i=k}^{k+1}\left|a_{i}^{j}-\omega_{i}^{j}\right|^{2} \leq C \sum_{j=1}^{N}\left\|\partial_{\xi} \widetilde{w}_{j}\right\|_{k}^{2}
$$

Step 4. We finally complete the desired estimates. Inserting (92), (93) and (95) into (90), we obtain

$$
\begin{aligned}
\sum_{j=1}^{N}\|w-P w\|_{I_{j}}^{2} & \leq \frac{h}{2} \sum_{j=1}^{N}\left\|\widetilde{w}_{j}-Q_{k} \widetilde{w}_{j}\right\|^{2}+C \frac{h}{2} \sum_{j=1}^{N}\left\|\partial_{\xi} \widetilde{w}_{j}\right\|_{k}^{2}, \\
\sum_{j=1}^{N}\left|(w-P w)_{j+1 / 2}\right|^{2} & \leq C \sum_{j=1}^{N}\left\|\partial_{\xi} \widetilde{w}_{j}\right\|_{k}^{2}, \\
\sum_{j=1}^{N}\|w-P w\|_{\infty, I_{j}}^{2} & \leq C\left(\sum_{j=1}^{N}\left\|\widetilde{w}_{j}-Q_{k} \widetilde{w}_{j}\right\|_{\infty}^{2}+\sum_{j=1}^{N}\left\|\partial_{\xi} \widetilde{w}_{j}\right\|_{k}^{2}\right) .
\end{aligned}
$$

Replacing $w$ in these inequalities by $w-v$, where $v$ is an arbitrary element in $\mathbb{V}_{h}^{k}$, and taking into account that $Q_{k} \widetilde{v}_{j}=\widetilde{v}_{j}$ gives

$$
\sum_{j=1}^{N}\|w-P w\|_{I_{j}}^{2} \leq \frac{h}{2} \sum_{j=1}^{N}\left\|\widetilde{w}_{j}-Q_{k} \widetilde{w}_{j}\right\|^{2}+C \frac{h}{2} \sum_{j=1}^{N}\left\|\partial_{\xi} \widetilde{w}_{j}-\partial_{\xi} \widetilde{v}_{j}\right\|_{k}^{2},
$$

(96b) $\sum_{j=1}^{N}\left|(w-P w)_{j+1 / 2}\right|^{2} \leq C \sum_{j=1}^{N}\left\|\partial_{\xi} \widetilde{w}_{j}-\partial_{\xi} \widetilde{v}_{j}\right\|_{k}^{2}$,

$$
\sum_{j=1}^{N}\|w-P w\|_{\infty, I_{j}}^{2} \leq C\left(\sum_{j=1}^{N}\left\|\widetilde{w}_{j}-Q_{k} \widetilde{w}_{j}\right\|_{\infty}^{2}+\sum_{j=1}^{N}\left\|\partial_{\xi} \widetilde{w}_{j}-\partial_{\xi} \widetilde{v}_{j}\right\|_{k}^{2}\right)
$$

The standard projection estimates give $\left\|\widetilde{w}_{j}-Q_{k} \widetilde{w}_{j}\right\| \leq C\left|\widetilde{w}_{j}\right|_{k+1}, \quad\left\|\widetilde{w}_{j}-Q_{k} \widetilde{w}_{j}\right\|_{\infty} \leq C h^{k+1 / 2}|w|_{k+1, I_{j}}=C h^{-1 / 2}\left|\widetilde{w}_{j}\right|_{k+1}$. 
By Theorem 3.1.1 in 13, there exists a constant $C$ such that

$$
\inf _{q \in P^{k-1}[-1,1]}\left\|\partial_{\xi} \widetilde{w}_{j}-q\right\|_{k} \leq C\left|\partial_{\xi} \widetilde{w}_{j}\right|_{k}=C\left|\widetilde{w}_{j}\right|_{k+1} .
$$

We now simply insert these estimates into (96) to conclude that

$$
\begin{aligned}
\sum_{j=1}^{N}\|w-P w\|_{I_{j}}^{2} & \leq C \frac{h}{2} \sum_{j=1}^{N}\left|\widetilde{w}_{j}\right|_{k+1}^{2}, \\
\sum_{j=1}^{N}\left|(w-P w)_{j+1 / 2}\right|^{2} & \leq C \sum_{j=1}^{N}\left|\widetilde{w}_{j}\right|_{k+1}^{2}, \\
\sum_{j=1}^{N}|w-P w|_{\infty, I_{j}}^{2} & \leq C \sum_{j=1}^{N}\left(h^{-1}\left|\widetilde{w}_{j}\right|_{k+1}^{2}+\left|\widetilde{w}_{j}\right|_{k+1}^{2}\right) \leq C h^{-1} \sum_{j=1}^{N}\left|\widetilde{w}_{j}\right|_{k+1}^{2}
\end{aligned}
$$

for any $k \geq 1$. The corresponding estimate at $x_{j-1 / 2}$ is obtained by symmetry. From these estimates we obtain by standard scaling the estimates as claimed.

In order to extend the above estimates to the multi-dimensional projection we need the following estimates which follow easily from the above analysis.

Corollary 7.2. Assume that $\left.w\right|_{I_{j}} \in H^{s+1}\left(I_{j}\right)$. Then we have

$$
\|P w\|^{2} \leq C \sum_{l=0}^{\min \{k, 2\}} h^{2 l} \sum_{j=1}^{N}|w|_{l, I_{j}}^{2}
$$

and

$$
\left\|\partial_{x}^{m}(w-P w)\right\|^{2} \leq C h^{2(p+1-m)} \sum_{j=1}^{N}|w|_{p+1, I_{j}}^{2}
$$

for any $1 \leq p \leq \min \{s, k\}$.

Proof. From (87) and (88) it follows that

$$
\|w\|^{2}=\sum_{j=1}^{N}\|w\|_{I_{j}}^{2}=\frac{h}{2} \sum_{j=1}^{N} \sum_{i=1}^{\infty}\left(\omega_{i}^{j}\right)^{2} \frac{2}{2 i-1}
$$

and

$$
\|P w\|^{2}=\sum_{j=1}^{N}\|P w\|_{I_{j}}^{2}=\frac{h}{2} \sum_{j=1}^{N}\left(\sum_{i=1}^{k+1}\left(\omega_{i}^{j}\right)^{2} \frac{2}{2-1}+\sum_{l=k}^{k+1}\left|a_{l}^{j}-\omega_{l}^{j}\right|^{2} \frac{2}{2 l-1}\right) .
$$

Hence using (95) we have

$$
\|P w\|^{2} \leq\|w\|^{2}+\frac{C h}{2} \sum_{j=1}^{N}\left\|\partial_{\xi} \widetilde{w}_{j}\right\|_{\min \{k, 2\}}^{2},
$$

which by a rescaling gives (97).

From (89) we have

$$
\begin{aligned}
\left.\partial_{x}^{m}(w-P w)\right|_{I_{j}}=\left(\partial _ { \xi } ^ { m } \left(\widetilde{w}_{j}\right.\right. & \left.-Q_{k} \widetilde{w}_{j}\right)+\left(\omega_{k}^{j}-a_{k}^{j}\right) \phi_{k}^{(m)}(\xi) \\
& \left.+\left(\omega_{k+1}^{j}-a_{k+1}^{j}\right) \phi_{k+1}^{(m)}(\xi)\right)\left(\frac{h}{2}\right)^{-m} .
\end{aligned}
$$


We continue as in the proof of Theorem 7.1 for the case $m=0$; finally, a standard scaling leads to (98).

Theorem 7.3. Let $P: H^{s+1} \rightarrow \mathbb{V}_{h}^{k}$ denote the one-dimensional global projection defined by (29) and denote by $\Pi=P^{(x)} \otimes P^{(y)}$ its tensor product along the two dimensions (or (69) with $d=2$ ). Then $\Pi$ is well defined on $\Omega$. If $w \in H^{s+1}(\Omega)$ for some $s \geq 1$, then

$$
\begin{aligned}
\|w-\Pi w\| & \leq C h^{p+1}|w|_{p+1, \Omega}, \\
\|w-\Pi w\|_{\Gamma_{h}} & \leq C h^{p+1 / 2}|w|_{p+1, \Omega}, \\
\|w-\Pi w\|_{\infty} & \leq C h^{p-1 / 2}|w|_{p+1, \Omega}
\end{aligned}
$$

for any $1 \leq p \leq \min \{s, k\}$.

Proof. By the definition of the tensor product, we see that $\left(P^{(y)} w\right)(x, y)$ is a polynomial of degree $k$ in $y$ with $x$-dependent coefficients that belong to $H^{s+1}\left(0, L_{x}\right)$; hence $\Pi w=P^{(x)}\left(P^{(y)} w\right)$ is well defined and also

$$
\Pi w=P^{(y)}\left(P^{(x)} w\right) .
$$

To prove the error estimate (100), we use (97), (98) through

$$
\|w-\Pi w\| \leq\left\|w-P^{(x)} w\right\|+\left\|P^{(x)}\left(w-P^{(y)} w\right)\right\| .
$$

We bound the first term using (98) with $m=0$ to obtain

$$
\left\|w-P^{(x)} w\right\|^{2} \leq C h^{2(p+1)} \sum \int_{K_{i j}}\left|\partial_{x}^{p+1} w\right|^{2} d x d y .
$$

Here and below $\sum$ denotes the sum over all $1 \leq i \leq N_{x}, 1 \leq j \leq N_{y}$, unless index is explicitly specified.

From (97) we have

$$
\left\|P^{(x)} w\right\|^{2} \leq C \sum_{l=0}^{\min \{k, 2\}} h^{2 l} \sum \int_{K_{i j}}\left|\partial_{x}^{l} w\right|^{2} d x d y,
$$

with which the second term is bounded by

$$
\begin{aligned}
\left\|P^{(x)}\left(w-P^{(y)} w\right)\right\|^{2} & \leq C \sum_{l=0}^{\min \{k, 2\}} h^{2 l} \sum \int_{K_{i j}}\left|\partial_{x}^{l}\left(w-P^{(y)} w\right)\right|^{2} d x d y \\
& =C \sum_{l=0}^{\min \{k, 2\}} h^{2 l} \sum \int_{K_{i j}}\left|\partial_{x}^{l} w-P^{(y)} \partial_{x}^{l} w\right|^{2} d y d x \\
& \leq C \sum_{l=0}^{\min \{k, 2\}} h^{2 l} h^{2(t+1)} \sum \int_{K_{i j}}\left|\partial_{y}^{t+1} \partial_{x}^{l} w\right|^{2} d x d y
\end{aligned}
$$

for any $0 \leq t \leq p-l$. Selecting $t=p-l$ gives

$$
\left\|P^{(x)}\left(w-P^{(y)} w\right)\right\|^{2} \leq C h^{2(p+1)} \sum_{l=0}^{\min \{k, 2\}} \sum \int_{K_{i j}}\left|\partial_{x}^{l} \partial_{y}^{p-l+1} w\right|^{2} d x d y .
$$

Thus (100a) follows. In order to prove (100b) we write

$$
\|w-\Pi w\|_{\Gamma_{h}}^{2}=\sum \int_{J_{j}}|w-\Pi w|_{x_{i+1 / 2}}^{2} d y+\sum \int_{I_{i}}|w-\Pi w|_{y_{j+1 / 2}}^{2} d x
$$


In virtue of (85) and (97), the first term on the right is bounded from above by

$$
\begin{aligned}
& 2 \sum \int_{J_{j}}\left|w-P^{(x)} w\right|_{x_{i+1 / 2}}^{2} d y+2 \sum \int_{J_{j}}\left|P^{(x)}\left(w-P^{(y)} w\right)\right|_{x_{i+1 / 2}}^{2} d y \\
& \leq 2 C h^{2 p+1} \sum \int_{K_{i j}}\left|\partial_{x}^{p+1} w\right|^{2} d x d y+2 C \sum \sum_{l=0}^{\min \{k, 2\}} h^{2 l} \int_{I_{i}}\left|\partial_{x}^{l}\left(w-P^{(y)} w\right)\right|_{y_{j+1} / 2}^{2} d x \\
& \leq 2 C h^{2 p+1} \sum \int_{K_{i j}}\left|\partial_{x}^{p+1} w\right|^{2} d x d y+2 C \sum \sum_{l=0}^{\min \{k, 2\}} h^{2 l} h^{2(t+1)} \int_{K_{i j}}\left|\partial_{y}^{t+1} \partial_{x}^{l} w\right|^{2} d x d y .
\end{aligned}
$$

Selecting $t=p-l$ gives

$$
\sum \int_{J_{j}}|w-\Pi w|_{x_{i+1 / 2}}^{2} d y \leq C h^{2 p+1}|w|_{p+1, \Omega}^{2}
$$

The second term in (101) shares this bound as well. Hence (100 b) is justified.

Finally we prove (100k), starting from

$$
\sum|w-\Pi w|_{\infty, K_{i j}}^{2} \leq 2 \sum\left|w-P^{(x)} w\right|_{\infty, K_{i j}}^{2}+2 \sum\left|P^{(x)}\left(w-P^{(y)} w\right)\right|_{\infty, K_{i j}}^{2}
$$

Rescaling the Sobolev estimate $|\widetilde{v}|_{\infty,[-1,1]}^{2} \leq 2\|\widetilde{v}\|_{1,[-1,1]}^{2}$ we have

$$
\sum_{j=1}^{N_{y}}|v(y)|_{\infty, J_{j}}^{2} h \leq 2 \sum_{l=0}^{1} h^{2 l}\left\|\partial_{y}^{l} v\right\|_{0,\left[0, L_{y}\right]}^{2} .
$$

Note that if $y \in \mathbb{R}^{d-1}$, the factor $h$ on the left should be $h^{d-1}$ and the summation index on the right runs up to $q>\frac{d-1}{2}$. With this, (866), and (98) with $m=0$, estimating the first term in (102) gives

$$
\begin{aligned}
\sum\left|w-P^{(x)} w\right|_{\infty, K_{i j}}^{2} & \leq 2 C h^{2 t_{1}} \sum \int_{I_{i}}\left|\partial_{x}^{t_{1}+1} w\right|_{\infty, J_{j}}^{2} d x \\
& \leq 2 C h^{2 t_{1}} \sum \int_{K_{i j}} h^{-1} \sum_{l=0}^{1} h^{2 l}\left|\partial_{y}^{l} \partial_{x}^{t_{1}+1} w\right|^{2} d x d y \\
& \leq C h^{2 p-1}|w|_{p+1, \Omega}^{2},
\end{aligned}
$$

where we have selected $t_{1}=p-l$.

Estimating the second term with (97) and (98) with $m=0$ yields

$$
\begin{aligned}
2 \sum\left|P^{(x)}\left(w-P^{(y)} w\right)\right|_{\infty, K_{i j}}^{2} & \leq 2 C \sum_{l=0}^{\min \{k, 2\}} h^{2 l} \int_{I_{i}}\left|\partial_{x}^{l}\left(w-P^{(y)} w\right)\right|_{\infty, J_{j}}^{2} d x \\
& =2 C \sum_{l=0}^{\min \{k, 2\}} h^{2 l} \int_{I_{i}}\left|\partial_{x}^{l} w-P^{(y)}\left(\partial_{x}^{l} w\right)\right|_{\infty, J_{j}}^{2} d x \\
& \leq 2 C \sum_{l=0}^{\min \{k, 2\}} h^{2 l} \sum \int_{K_{i j}} h^{2 t}\left|\partial_{y}^{t+1} \partial_{x}^{l} w\right|^{2} d x d y \\
& \leq C h^{2 p}|w|_{p+1, \Omega}^{2},
\end{aligned}
$$

where we have selected $t=p-l$. 
Therefore,

$$
\sum|w-\Pi w|_{\infty, K_{i j}}^{2} \leq C h^{2 p-1}|w|_{p+1, \Omega}^{2} .
$$

Remark 7.1. Note that the above analysis when applied to the $d$-dimensional case yields the following estimate:

$$
\sum|w-\Pi w|_{\infty, K_{\alpha}}^{2} \leq C h^{2 p+1-d}|w|_{p+1, \Omega}^{2} .
$$

Hence, for $d>2$, (100 ) needs to be replaced by

$$
\|w-\Pi w\|_{\infty} \leq C h^{p+1 / 2-d / 2}|w|_{p+1, \Omega},
$$

while $(100$ ) and $(100 \mathrm{~b})$ remain valid; these together lead to (71) as needed.

\section{ACKNOWLEDGMENTS}

The author wishes to thank the referees for a careful reading of the manuscript and valuable suggestions resulting in improvements to this paper. This research was partially supported by the National Science Foundation under Grants DMS0907963, DMS13-12636, DMS1312636 and by NSF Grant RNMS (Ki-Net) 1107291.

\section{REFERENCES}

[1] D. N. Arnold, An interior penalty finite element method with discontinuous elements, SIAM J. Numer. Anal. 19 (1982), no. 4, 742-760, DOI 10.1137/0719052. MR664882 (83f:65173)

[2] D. N. Arnold, F. Brezzi, B. Cockburn, and L. D. Marini, Unified analysis of discontinuous Galerkin methods for elliptic problems, SIAM J. Numer. Anal. 39 (2001/02), no. 5, 17491779, DOI 10.1137/S0036142901384162. MR.1885715(2002k:65183)

[3] G. A. Baker, Finite element methods for elliptic equations using nonconforming elements, Math. Comp. 31 (1977), no. 137, 45-59. MR0431742 (55 \#4737)

[4] J. L. Bona, H. Chen, O. Karakashian, and Y. Xing, Conservative, discontinuous Galerkinmethods for the generalized Korteweg-de Vries equation, Math. Comp. 82 (2013), no. 283, 1401-1432, DOI 10.1090/S0025-5718-2013-02661-0. MR3042569

[5] F. Bassi and S. Rebay, A high-order accurate discontinuous finite element method for the numerical solution of the compressible Navier-Stokes equations, J. Comput. Phys. 131 (1997), no. 2, 267-279, DOI 10.1006/jcph.1996.5572. MR 1433934 (97m:76078)

[6] C. E. Baumann and J. T. Oden, A discontinuous hp finite element method for convectiondiffusion problems, Comput. Methods Appl. Mech. Engrg. 175 (1999), no. 3-4, 311-341, DOI 10.1016/S0045-7825(98)00359-4. MR:1702201(2000d:65171)

[7] P. Castillo, An optimal estimate for the local discontinuous Galerkin method, Discontinuous Galerkin Methods (1999), Lect. Notes Comput. Sci. Eng., vol. 11, Springer, Berlin, 2000, pp. 285-290, DOI 10.1007/978-3-642-59721-3_23. MR1842183(2002d:65096)

[8] F. Celiker and B. Cockburn, Superconvergence of the numerical traces of discontinuous Galerkin and hybridized methods for convection-diffusion problems in one space dimension, Math. Comp. 76 (2007), no. 257, 67-96, DOI 10.1090/S0025-5718-06-01895-3. MR2261012 (2008e:65225)

[9] B. Cockburn, G. Kanschat, I. Perugia, and D. Schötzau, Superconvergence of the local discontinuous Galerkin method for elliptic problems on Cartesian grids, SIAM J. Numer. Anal. 39 (2001), no. 1, 264-285 (electronic), DOI 10.1137/S0036142900371544. MR1860725 (2002g:65140)

[10] P. Castillo, B. Cockburn, I. Perugia, and D. Schötzau, An a priori error analysis of the local discontinuous Galerkin method for elliptic problems, SIAM J. Numer. Anal. 38 (2000), no. 5, 1676-1706 (electronic), DOI 10.1137/S0036142900371003. MR1813251(2002k:65175)

[11] P. Castillo, B. Cockburn, D. Schötzau, and C. Schwab, Optimal a priori error estimates for the hp-version of the local discontinuous Galerkin method for convection-diffusion problems, Math. Comp. 71 (2002), no. 238, 455-478, DOI 10.1090/S0025-5718-01-01317-5. MR.1885610 (2003e:65214) 
[12] Y. Cheng and C.-W. Shu, A discontinuous Galerkin finite element method for time dependent partial differential equations with higher order derivatives, Math. Comp. 77 (2008), no. 262, 699-730, DOI 10.1090/S0025-5718-07-02045-5. MR2373176 (2008m:65252)

[13] P. G. Ciarlet, The Finite Element Method for Elliptic Problems, North-Holland Publishing Co., Amsterdam, 1978. Studies in Mathematics and its Applications, Vol. 4. MR0520174 (58 \#25001)

[14] B. Cockburn and C. Dawson, Approximation of the velocity by coupling discontinuous Galerkin and mixed finite element methods for flow problems, Comput. Geosci. 6 (2002), no. 3-4, 505-522, DOI 10.1023/A:1021203618109. Locally conservative numerical methods for flow in porous media. MR 1956028(2004b:65179)

[15] B. Cockburn, S. Hou, and C.-W. Shu, The Runge-Kutta local projection discontinuous Galerkin finite element method for conservation laws. IV. The multidimensional case, Math. Comp. 54 (1990), no. 190, 545-581, DOI 10.2307/2008501. MR1010597 (90k:65162)

[16] B. Cockburn, G. Kanschat, and D. Schotzau, A locally conservative LDG method for the incompressible Navier-Stokes equations, Math. Comp. 74 (2005), no. 251, 1067-1095 (electronic), DOI 10.1090/S0025-5718-04-01718-1. MR2136994(2006a:65157)

[17] B. Cockburn, S. Y. Lin, and C.-W. Shu, TVB Runge-Kutta local projection discontinuous Galerkin finite element method for conservation laws. III. One-dimensional systems, J. Comput. Phys. 84 (1989), no. 1, 90-113, DOI 10.1016/0021-9991(89)90183-6. MR.1015355 (90k:65161)

[18] B. Cockburn and C.-W. Shu, TVB Runge-Kutta local projection discontinuous Galerkin finite element method for conservation laws. II. General framework, Math. Comp. 52 (1989), no. 186, 411-435, DOI 10.2307/2008474. MR983311 (90k:65160)

[19] B. Cockburn and C.-W. Shu, The local discontinuous Galerkin method for time-dependent convection-diffusion systems, SIAM J. Numer. Anal. 35 (1998), no. 6, 2440-2463 (electronic), DOI 10.1137/S0036142997316712. MR 1655854 (99j:65163)

[20] Y. Cheng and C.-W. Shu, A discontinuous Galerkin finite element method for time dependent partial differential equations with higher order derivatives, Math. Comp. 77 (2008), no. 262, 699-730, DOI 10.1090/S0025-5718-07-02045-5. MR2373176 (2008m:65252)

[21] B. Cockburn and C.-W. Shu, The Runge-Kutta discontinuous Galerkin method for conservation laws. V. Multidimensional systems, J. Comput. Phys. 141 (1998), no. 2, 199-224, DOI 10.1006/jcph.1998.5892. MR.1619652(99c:65181)

[22] G. Gassner, F. Lörcher, and C.-D. Munz, A contribution to the construction of diffusion fluxes for finite volume and discontinuous Galerkin schemes, J. Comput. Phys. 224 (2007), no. 2, 1049-1063, DOI 10.1016/j.jcp.2006.11.004. MR.2330305 (2008b:76128)

[23] Y. Huang, H. Liu, and N. Yi, Recovery of normal derivatives from the piecewise $L^{2}$ projection, J. Comput. Phys. 231 (2012), no. 4, 1230-1243, DOI 10.1016/j.jcp.2011.10.001. MR2876452

[24] C. Johnson and J. Pitkäranta, An analysis of the discontinuous Galerkin method for a scalar hyperbolic equation, Math. Comp. 46 (1986), no. 173, 1-26, DOI 10.2307/2008211. MR815828 (88b:65109)

[25] B. van Leer and S. Nomura, Discontinuous Galerkin for Diffusion, Proceedings of 17th AIAA Computational Fluid Dynamics Conference (June 6 2005), AIAA-2005-5108.

[26] P. Lasaint and P.-A. Raviart, On a finite element method for solving the neutron transport equation, Mathematical Aspects of Finite Elements in Partial Differential Equations (Proc. Sympos., Math. Res. Center, Univ. Wisconsin, Madison, Wis., 1974), Math. Res. Center, Univ. of Wisconsin-Madison, Academic Press, New York, 1974, pp. 89-123. Publication No. 33. MR0658142(58 \#31918)

[27] H. Liu and J. Yan, The direct discontinuous Galerkin (DDG) methods for diffusion problems, SIAM J. Numer. Anal. 47 (2008/09), no. 1, 675-698, DOI 10.1137/080720255. MR2475957 (2010b:65207)

[28] H. Liu and J. Yan, The direct discontinuous Galerkin (DDG) method for diffusion with interface corrections, Commun. Comput. Phys. 8 (2010), no. 3, 541-564, DOI 10.4208/cicp.010909.011209a. MR2673775 (2011g:65196)

[29] J. T. Oden, I. Babuška, and C. E. Baumann, A discontinuous hp finite element method for diffusion problems, J. Comput. Phys. 146 (1998), no. 2, 491-519, DOI 10.1006/jcph.1998.6032. MR:1654911(99m:65173) 
[30] T. E. Peterson, A note on the convergence of the discontinuous Galerkin method for a scalar hyperbolic equation, SIAM J. Numer. Anal. 28 (1991), no. 1, 133-140, DOI 10.1137/0728006. MR.1083327 (91m:65250)

[31] W. H. Reed and T. R. Hill, Triangular mesh methods for the neutron transport equation, Technical Report Tech. Report LA-UR-73-479, Los Alamos Scientific Laboratory, 1973.

[32] G. R. Richter, An optimal-order error estimate for the discontinuous Galerkin method, Math. Comp. 50 (1988), no. 181, 75-88, DOI 10.2307/2007915. MR917819 (88j:65197)

[33] B. Rivière and M. F. Wheeler, A discontinuous Galerkin method applied to nonlinear parabolic equations, Discontinuous Galerkin methods (1999), Lect. Notes Comput. Sci. Eng., vol. 11, Springer, Berlin, 2000, pp. 231-244, DOI 10.1007/978-3-642-59721-3_17. MR.1842177 (2002d:65105)

[34] Ch. Schwab, p- and hp-Finite Element Methods, Numerical Mathematics and Scientific Computation, The Clarendon Press Oxford University Press, New York, 1998. Theory and applications in solid and fluid mechanics. MR1695813 (2000d:65003)

[35] M. F. Wheeler, An elliptic collocation-finite element method with interior penalties, SIAM J. Numer. Anal. 15 (1978), no. 1, 152-161. MR0471383 (57 \#11117)

[36] Y. Xu and C.-W. Shu, Error estimates of the semi-discrete local discontinuous Galerkin method for nonlinear convection-diffusion and KdV equations, Comput. Methods Appl. Mech. Engrg. 196 (2007), no. 37-40, 3805-3822, DOI 10.1016/j.cma.2006.10.043. MR2340006 (2009e:65139)

[37] Y. Xu and C.-W. Shu, Optimal error estimates of the semidiscrete local discontinuous Galerkin methods for high order wave equations, SIAM J. Numer. Anal. 50 (2012), no. 1, 79-104, DOI 10.1137/11082258X. MR2888305

[38] J. Yan and C.-W. Shu, A local discontinuous Galerkin method for KdV type equations, SIAM J. Numer. Anal. 40 (2002), no. 2, 769-791 (electronic), DOI 10.1137/S0036142901390378. MR.1921677(2003e:65181)

[39] M. Zhang and J. Yan, Fourier type error analysis of the direct discontinuous Galerkin method and its variations for diffusion equations, J. Sci. Comput. 52 (2012), no. 3, 638-655, DOI 10.1007/s10915-011-9564-5. MR2948711

[40] Q. Zhang and C.-W. Shu, Error estimates to smooth solutions of Runge-Kutta discontinuous Galerkin methods for scalar conservation laws, SIAM J. Numer. Anal. 42 (2004), no. 2, 641-666 (electronic), DOI 10.1137/S0036142902404182. MR2084230 (2005h:65149)

[41] Q. Zhang and C.-W. Shu, Error estimates to smooth solutions of Runge-Kutta discontinuous Galerkin method for symmetrizable systems of conservation laws, SIAM J. Numer. Anal. 44 (2006), no. 4, 1703-1720 (electronic), DOI 10.1137/040620382. MR2257123(2008f:65162)

[42] S. Osher, Riemann solvers, the entropy condition, and difference approximations, SIAM J. Numer. Anal. 21 (1984), no. 2, 217-235, DOI 10.1137/0721016. MR736327 (86d:65119)

Department of Mathematics, Iowa State University, Ames, Iowa 50010

E-mail address: hliu@iastate.edu 